\title{
Feller semigroups and degenerate elliptic operators with Wentzell boundary conditions
}

\author{
by \\ Kazuaki Taira (Tsukuba), Angelo Favini (Bologna) and \\ Silvia Romanelli (Bari)
}

\begin{abstract}
This paper is devoted to the functional analytic approach to the problem of construction of Feller semigroups with Wentzell boundary conditions in the characteristic case. Our results may be stated as follows: We can construct Feller semigroups corresponding to a diffusion phenomenon including absorption, reflection, viscosity, diffusion along the boundary and jump at each point of the boundary.
\end{abstract}

1. Introduction and results. Let $D$ be a bounded domain in Euclidean space $\mathbb{R}^{N}, N \geq 2$, with smooth boundary $\partial D$; its closure $\bar{D}=D \cup \partial D$ is an $N$-dimensional, compact smooth manifold with boundary (see Figure 1.1).

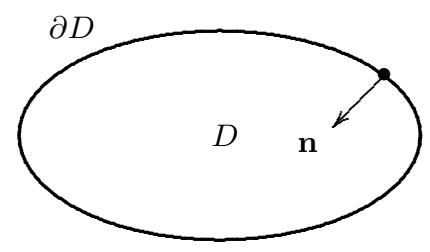

Fig. 1.1

Let $C(\bar{D})$ be the space of all continuous real-valued functions on $\bar{D}$. We equip the space $C(\bar{D})$ with the topology of uniform convergence on the whole $\bar{D}$; hence it is a Banach space with the maximum norm

$$
\|f\|_{\infty}=\max _{x \in \bar{D}}|f(x)|, \quad f \in C(\bar{D}) .
$$

2000 Mathematics Subject Classification: Primary 47D07, 35J25; Secondary 47D06, 60J35, $60 \mathrm{~J} 60$.

Key words and phrases: Feller semigroup, boundary value problem, characteristic case.

Work partially supported by M.U.R.S.T. $60 \%$ and $40 \%$ under the auspices of G.N.A.F.A. of C.N.R. 
A strongly continuous semigroup $\left\{T_{t}\right\}_{t>0}$ on $C(\bar{D})$ is called a Feller semigroup on $\bar{D}$ if it is non-negative and contractive on $C(\bar{D})$ :

$$
f \in C(\bar{D}), 0 \leq f(x) \leq 1 \text { on } \bar{D} \Rightarrow 0 \leq T_{t} f(x) \leq 1 \text { on } \bar{D} .
$$

It is known (see [5], [16]) that if $T_{t}$ is a Feller semigroup on $\bar{D}$, then there exists a unique Markov transition function $p_{t}(x, \cdot)$ on $\bar{D}$ such that

$$
T_{t} f(x)=\int_{\bar{D}} p_{t}(x, d y) f(y), \quad f \in C(\bar{D}) .
$$

Furthermore it can be shown that the function $p_{t}(x, \cdot)$ is the transition function of some strong Markov process; hence the value $p_{t}(x, E)$ expresses the transition probability that a Markovian particle starting at position $x$ will be found in the set $E$ at time $t$.

Let $A$ be a second-order, degenerate elliptic differential operator with real coefficients such that

$$
A u(x)=\sum_{i, j=1}^{N} a^{i j}(x) \frac{\partial^{2} u}{\partial x_{i} \partial x_{j}}(x)+\sum_{i=1}^{N} b^{i}(x) \frac{\partial u}{\partial x_{i}}(x)+c(x) u(x),
$$

where:

(1) $a^{i j} \in C^{\infty}\left(\mathbb{R}^{N}\right), a^{i j}(x)=a^{j i}(x)$ and

$$
\sum_{i, j=1}^{N} a^{i j}(x) \xi_{i} \xi_{j} \geq 0, \quad x \in \mathbb{R}^{N}, \xi \in \mathbb{R}^{N}
$$

(2) $b^{i} \in C^{\infty}\left(\mathbb{R}^{N}\right)$.

(3) $c \in C^{\infty}\left(\mathbb{R}^{N}\right)$ and $c(x) \leq 0$ on $\bar{D}$.

The functions $a^{i j}(x), b^{i}(x)$ and $c(x)$ are called the diffusion coefficients, the drift coefficients and the termination coefficient, respectively.

In this paper we study the case where the operator $A$ is characteristic with respect to the boundary $\partial D$, which we formulate precisely.

Following Fichera [8], we introduce a function $b\left(x^{\prime}\right)$ on the boundary $\partial D$ by the formula

$$
b\left(x^{\prime}\right)=\sum_{i=1}^{N}\left(b^{i}\left(x^{\prime}\right)-\sum_{j=1}^{N} \frac{\partial a^{i j}}{\partial x_{j}}\left(x^{\prime}\right)\right) n_{i}, \quad x^{\prime} \in \partial D,
$$

where $\mathbf{n}=\left(n_{1}, \ldots, n_{N}\right)$ is the unit interior normal to $\partial D$ at $x^{\prime}$ (see Figure 1.1). The function $b\left(x^{\prime}\right)$ will be called the Fichera function for the operator $A$. We divide the boundary $\partial D$ into the following four disjoint subsets 
(see [8], [13], [15]):

$$
\begin{aligned}
& \Sigma_{3}=\left\{x^{\prime} \in \partial D: \sum_{i, j=1}^{N} a^{i j}\left(x^{\prime}\right) n_{i} n_{j}>0\right\}, \\
& \Sigma_{2}=\left\{x^{\prime} \in \partial D: \sum_{i, j=1}^{N} a^{i j}\left(x^{\prime}\right) n_{i} n_{j}=0, b\left(x^{\prime}\right)<0\right\}, \\
& \Sigma_{1}=\left\{x^{\prime} \in \partial D: \sum_{i, j=1}^{N} a^{i j}\left(x^{\prime}\right) n_{i} n_{j}=0, b\left(x^{\prime}\right)>0\right\}, \\
& \Sigma_{0}=\left\{x^{\prime} \in \partial D: \sum_{i, j}^{N} a^{i j}\left(x^{\prime}\right) n_{i} n_{j}=0, b\left(x^{\prime}\right)=0\right\} .
\end{aligned}
$$

It is worth pointing out (see [13], [15]) that one may impose a boundary condition only on the set $\Sigma_{2} \cup \Sigma_{3}$, since a Markovian particle reaches the boundary $\partial D$ by means of the diffusion vector fields

$$
\pm X_{i}(x)= \pm \sum_{j=1}^{N} a^{i j}(x) \frac{\partial}{\partial x_{j}}, \quad 1 \leq i \leq N,
$$

and the drift vector field

$$
X_{0}\left(x^{\prime}\right)=\sum_{i=1}^{N}\left(b^{i}\left(x^{\prime}\right)-\sum_{j=1}^{N} \frac{\partial a^{i j}}{\partial x_{j}}\left(x^{\prime}\right)\right) \frac{\partial}{\partial x_{i}},
$$

and

$$
b\left(x^{\prime}\right)=\left\langle X_{0}\left(x^{\prime}\right), \mathbf{n}\right\rangle .
$$

Moreover, in the one-dimensional case $(N=1)$ the four sets $\Sigma_{3}, \Sigma_{2}, \Sigma_{1}$ and $\Sigma_{0}$ are supposed to correspond to a regular boundary, an exit boundary, an entrance boundary and a natural boundary, respectively (see [6]).

The fundamental hypothesis for $A$ is the following (see Figure 1.2):

(H) $\quad \partial D=\Sigma_{0} \cup \Sigma_{1} \cup \Sigma_{2}$ and each set $\Sigma_{i}(i=0,1,2)$ consists of a finite number of connected hypersurfaces.

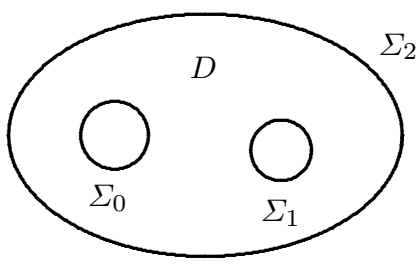

Fig. 1.2 
ExAmple. Let $D$ be the unit open disk in $\mathbb{R}^{2}(N=2)$. Assume that, in terms of polar coordinates $(r, \theta)$, the differential operator $A$ is written in the form

$$
A=\varphi(r) \Delta+\frac{\partial}{\partial r}=\varphi(r)\left(\frac{\partial^{2}}{\partial r^{2}}+\frac{1}{r} \frac{\partial}{\partial r}+\frac{1}{r^{2}} \frac{\partial^{2}}{\partial \theta^{2}}\right)+\frac{\partial}{\partial r},
$$

where $\varphi(r)$ is a smooth function defined by the formula

$$
\varphi(r)= \begin{cases}\exp \left[-1 /\left(1-r^{2}\right)\right] & \text { for } r<1 \\ 0 & \text { for } r \geq 1\end{cases}
$$

Then it is easy to see that $\Sigma_{3}=\emptyset, b=-1$ on $\partial D$, and thus $\partial D=\Sigma_{2}$.

Let $L$ be a second-order boundary condition such that, in local coordinates $\left(x_{1}, \ldots, x_{N-1}\right)$ on $\Sigma_{2}$,

$$
\begin{aligned}
L u\left(x^{\prime}\right)= & \sum_{i, j=1}^{N-1} \alpha^{i j}\left(x^{\prime}\right) \frac{\partial^{2} u}{\partial x_{i} \partial x_{j}}\left(x^{\prime}\right)+\sum_{i=1}^{N-1} \beta^{i}\left(x^{\prime}\right) \frac{\partial u}{\partial x_{i}}\left(x^{\prime}\right) \\
& +\gamma\left(x^{\prime}\right) u\left(x^{\prime}\right)+\mu\left(x^{\prime}\right) \frac{\partial u}{\partial \mathbf{n}}\left(x^{\prime}\right)-\delta\left(x^{\prime}\right) A u\left(x^{\prime}\right) \\
& +\int_{\Sigma_{2}} r\left(x^{\prime}, y^{\prime}\right)\left[u\left(y^{\prime}\right)-u\left(x^{\prime}\right)-\sum_{j=1}^{N-1}\left(y_{j}-x_{j}\right) \frac{\partial u}{\partial x_{j}}\left(x^{\prime}\right)\right] d y^{\prime},
\end{aligned}
$$

where:

(1) The $\alpha^{i j}$ are the components of a smooth symmetric contravariant tensor of type $\left(\begin{array}{l}2 \\ 0\end{array}\right)$ on $\Sigma_{2}$ and

$$
\sum_{i, j=1}^{N-1} \alpha^{i j}\left(x^{\prime}\right) \eta_{i} \eta_{j} \geq 0, \quad x^{\prime} \in \Sigma_{2}, \quad \eta=\sum_{j=1}^{N-1} \eta_{j} d x_{j} \in T_{x^{\prime}}^{*}\left(\Sigma_{2}\right) .
$$

Here $T_{x^{\prime}}^{*}\left(\Sigma_{2}\right)$ is the cotangent space of $\Sigma_{2}$ at $x^{\prime}$.

(2) $\beta^{i} \in C^{\infty}\left(\Sigma_{2}\right)$.

(3) $\gamma \in C^{\infty}\left(\Sigma_{2}\right)$ and $\gamma\left(x^{\prime}\right) \leq 0$ on $\Sigma_{2}$.

(4) $\mu \in C^{\infty}\left(\Sigma_{2}\right)$ and $\mu\left(x^{\prime}\right) \geq 0$ on $\Sigma_{2}$.

(5) $\delta \in C^{\infty}\left(\Sigma_{2}\right)$ and $\delta\left(x^{\prime}\right) \geq 0$ on $\Sigma_{2}$.

(6) $\mathbf{n}=\left(n_{1}, \ldots, n_{N}\right)$ is the unit interior normal to $\Sigma_{2}$.

(7) The integrand kernel $r\left(x^{\prime}, y^{\prime}\right)$ is the distribution kernel of a properly supported pseudo-differential operator $R \in L_{1,0}^{2-k}\left(\Sigma_{2}\right), k>0$, and it is nonnegative off the diagonal $\Delta_{\Sigma_{2}}=\left\{\left(x^{\prime}, x^{\prime}\right): x^{\prime} \in \Sigma_{2}\right\}$ in $\Sigma_{2} \times \Sigma_{2}$. The density $d y^{\prime}$ is a strictly positive density on $\Sigma_{2}$. 
The boundary condition $L$ is called a second-order Wentzell boundary condition (see [22]). The five terms of $L$

$$
\begin{aligned}
& \sum_{i, j=1}^{N-1} \alpha^{i j}\left(x^{\prime}\right) \frac{\partial^{2} u}{\partial x_{i} \partial x_{j}}\left(x^{\prime}\right)+\sum_{i=1}^{N-1} \beta^{i}\left(x^{\prime}\right) \frac{\partial u}{\partial x_{i}}\left(x^{\prime}\right), \\
& \gamma\left(x^{\prime}\right) u\left(x^{\prime}\right), \quad \mu\left(x^{\prime}\right) \frac{\partial u}{\partial \mathbf{n}}\left(x^{\prime}\right), \quad \delta\left(x^{\prime}\right) A u\left(x^{\prime}\right), \\
& \int_{\Sigma_{2}} r\left(x^{\prime}, y^{\prime}\right)\left[u\left(y^{\prime}\right)-u\left(x^{\prime}\right)-\sum_{j=1}^{N-1}\left(y_{j}-x_{j}\right) \frac{\partial u}{\partial x_{j}}\left(x^{\prime}\right)\right] d y^{\prime}
\end{aligned}
$$

are supposed to correspond to diffusion along $\Sigma_{2}$, absorption, reflection, viscosity and jump on $\Sigma_{2}$, respectively (see Figures $1.3,1.4$ and 1.5).

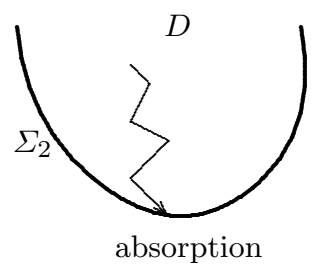

Fig. 1.3

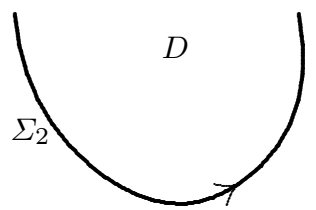

diffusion along the boundary
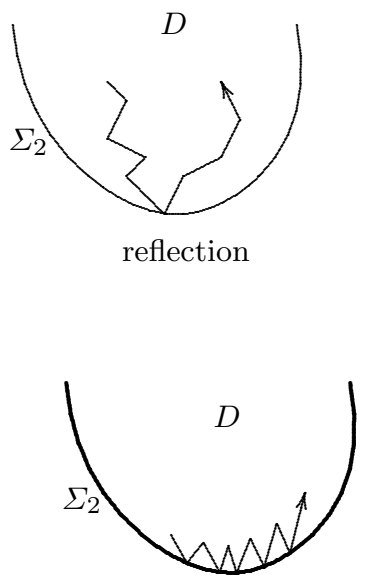

viscosity

Fig. 1.4

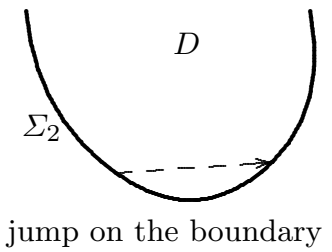

Fig. 1.5

We say that the boundary condition $L$ is transversal on the set $\Sigma_{2}$ if it satisfies the condition

$$
\mu\left(x^{\prime}\right)+\delta\left(x^{\prime}\right)>0, \quad x^{\prime} \in \Sigma_{2} .
$$


Intuitively, the transversality condition implies that either reflection or viscosity occurs on $\Sigma_{2}$. Probabilistically, this means that every Markov process on $\Sigma_{2}$ is the "trace" on $\Sigma_{2}$ of trajectories of some Markov process on the closure $\bar{D}=D \cup \partial D$ (see Figure 1.6).

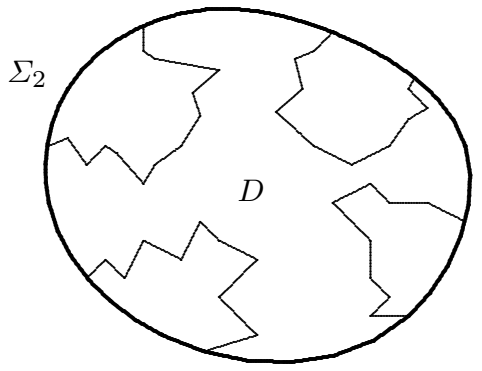

Fig. 1.6

It is known (see [1], [14], [16], [22]) that the infinitesimal generator $\mathfrak{A}$ of a Feller semigroup $\left\{T_{t}\right\}_{t \geq 0}$ is described analytically by a degenerate elliptic operator $A$ and a Wentzell boundary condition $L$.

This paper is devoted to the functional analytic approach to the problem of construction of Feller semigroups with Wentzell boundary conditions. More precisely we prove that there exists a Feller semigroup on $\bar{D}$ corresponding to a diffusion phenomenon including absorption, reflection, viscosity, diffusion along $\Sigma_{2}$ and jump on $\Sigma_{2}$, both in the transversal and non-transversal cases.

First we consider the transversal case:

TheOREm 1.1. Assume that the operator A satisfies hypothesis $(\mathrm{H})$ and that the boundary condition $L$ is transversal on $\Sigma_{2}$. Then there exists a Feller semigroup $\left\{T_{t}\right\}_{t \geq 0}$ on $\bar{D}$ whose infinitesimal generator $\mathfrak{A}$ is defined by $\mathfrak{A} u=A u$ for $u \in \mathcal{D}(\mathfrak{A})$, where

$$
\mathcal{D}(\mathfrak{A})=\left\{u \in C(\bar{D}): A u \in C(\bar{D}), L u=0 \text { on } \Sigma_{2}\right\} .
$$

Here $A u$ and $L u$ are taken in the sense of distributions. Furthermore the generator $\mathfrak{A}$ coincides with the minimal closed extension in $C(\bar{D})$ of the restriction of $A$ to the space $\left\{u \in C^{\infty}(\bar{D}): L u=0\right.$ on $\left.\Sigma_{2}\right\}$.

We remark that Taira [18] proved Theorem 1.1 assuming that the transversal condition holds on the set $\Sigma_{2} \cup \Sigma_{3}$, but $L$ does not contain an integral term corresponding to the jump phenomenon on $\Sigma_{2}$, while Cattiaux [3] obtained a probabilistic version of Theorem 1.1 in the non-characteristic case: $\partial D=\Sigma_{3}$.

Next we generalize Theorem 1.1 to the non-transversal case. To this end, we assume that there exists a second order Wentzell boundary condition $L_{\nu}$ 
such that

$$
L u\left(x^{\prime}\right)=m\left(x^{\prime}\right) L_{\nu} u\left(x^{\prime}\right)+\gamma\left(x^{\prime}\right) u\left(x^{\prime}\right) \quad \text { on } \Sigma_{2},
$$

where

$$
m \in C^{\infty}\left(\Sigma_{2}\right) \text { and } m\left(x^{\prime}\right) \geq 0 \text { on } \Sigma_{2}
$$

and the boundary condition $L_{\nu}$ is given, in local coordinates $\left(x_{1}, \ldots, x_{N-1}\right)$ on $\Sigma_{2}$, by the formula

$$
\begin{aligned}
L_{\nu} u\left(x^{\prime}\right):= & \sum_{i, j=1}^{N-1} \bar{\alpha}^{i j}\left(x^{\prime}\right) \frac{\partial^{2} u}{\partial x_{i} \partial x_{j}}\left(x^{\prime}\right)+\sum_{i=1}^{N-1} \bar{\beta}^{i}\left(x^{\prime}\right) \frac{\partial u}{\partial x_{i}}\left(x^{\prime}\right) \\
& +\bar{\mu}\left(x^{\prime}\right) \frac{\partial u}{\partial \mathbf{n}}\left(x^{\prime}\right)-\bar{\delta}\left(x^{\prime}\right) A u\left(x^{\prime}\right) \\
& +\int_{\Sigma_{2}} \bar{r}\left(x^{\prime}, y^{\prime}\right)\left[u\left(y^{\prime}\right)-u\left(x^{\prime}\right)-\sum_{j=1}^{N-1}\left(y_{j}-x_{j}\right) \frac{\partial u}{\partial x_{j}}\left(x^{\prime}\right)\right] d y^{\prime}
\end{aligned}
$$

and satisfies the transversality condition

$$
\bar{\mu}\left(x^{\prime}\right)+\bar{\delta}\left(x^{\prime}\right)>0 \quad \text { on } \Sigma_{2} .
$$

Observe that, since

$$
\mu\left(x^{\prime}\right)=m\left(x^{\prime}\right) \bar{\mu}\left(x^{\prime}\right) \quad \text { and } \quad \delta\left(x^{\prime}\right)=m\left(x^{\prime}\right) \bar{\delta}\left(x^{\prime}\right),
$$

the boundary condition $L$ is not transversal on $\Sigma_{2}$. Moreover we assume that

$$
m\left(x^{\prime}\right)-\gamma\left(x^{\prime}\right)>0 \quad \text { on } \Sigma_{2} .
$$

The intuitive meaning of conditions (A) and (B) is that a Markovian particle does not stay on $\Sigma_{2}$ for any period of time until it "dies" when reaching the set

$$
M=\left\{x^{\prime} \in \Sigma_{2}: m\left(x^{\prime}\right)=0\right\},
$$

where the particle is definitively absorbed.

Now we introduce a subspace of $C(\bar{D})$ which is associated with the boundary condition $L$. By (B), the boundary condition

$$
L u\left(x^{\prime}\right)=m\left(x^{\prime}\right) L_{\nu} u\left(x^{\prime}\right)+\gamma\left(x^{\prime}\right) u\left(x^{\prime}\right)=0 \quad \text { on } \Sigma_{2}
$$

includes the condition

$$
u\left(x^{\prime}\right)=0 \quad \text { on } M .
$$

With this fact in mind, we let

$$
C_{0}(\bar{D} \backslash M)=\left\{u \in C(\bar{D}): u\left(x^{\prime}\right)=0 \text { on } M\right\} .
$$

This is a closed subspace of $C(\bar{D})$; hence it is a Banach space. 
A strongly continuous semigroup $\left\{U_{t}\right\}_{t \geq 0}$ on the space $C_{0}(\bar{D} \backslash M)$ is called a Feller semigroup on $\bar{D} \backslash M$ if it is non-negative and contractive on $C_{0}(\bar{D} \backslash M)$ :

$$
f \in C_{0}(\bar{D} \backslash M), 0 \leq f(x) \leq 1 \text { on } \bar{D} \backslash M \Rightarrow 0 \leq U_{t} f(x) \leq 1 \text { on } \bar{D} \backslash M .
$$

The next theorem is a generalization of Theorem 1.1 to the non-transversal case:

Theorem 1.2. Assume that the differential operator A satisfies condition $(\mathrm{H})$ and the boundary operator $L$ satisfies conditions $(\mathrm{A}),\left(\mathrm{A}_{1}\right),\left(\mathrm{A}_{2}\right)$, $(\overline{\mathrm{T}})$ and $(\mathrm{B})$. Define a linear operator $\mathfrak{A}_{0}: C_{0}(\bar{D} \backslash M) \rightarrow C_{0}(\bar{D} \backslash M)$ as follows: $\mathfrak{A}_{0} u=A u$ for $u \in \mathcal{D}\left(\mathfrak{A}_{0}\right)$, where

$$
\mathcal{D}\left(\mathfrak{A}_{0}\right)=\left\{u \in C_{0}(\bar{D} \backslash M): A u \in C_{0}(\bar{D} \backslash M), \text { Lu }=0 \text { on } \Sigma_{2}\right\} .
$$

Then the operator $\mathfrak{A}_{0}$ generates a Feller semigroup $\left\{U_{t}\right\}_{t \geq 0}$ on $C_{0}(\bar{D} \backslash M)$.

Theorem 1.2 asserts that there exists a Feller semigroup on $\bar{D} \backslash M$ corresponding to the following diffusion phenomenon: a Markovian particle moves both by jumps and continuously in the state space $\bar{D} \backslash M$ until it "dies" when reaching the set $M$ where it is definitively absorbed.

The rest of this paper is organized as follows.

In Section 2 we present a brief description of the basic definitions and results about a class of semigroups (Feller semigroups) associated with Markov processes in probability theory, which forms a functional analytic background for the proof of Theorems 1.1 and 1.2.

Section 3 provides a review of the basic concepts and results of the theory of pseudo-differential operators which will be used in the subsequent sections. In particular we give an existence and uniqueness theorem for a class of pseudo-differential operators (Theorem 3.1), essentially due to Cancelier [2], which enters naturally in the construction of Feller semigroups.

In Section 4 we prove a general existence theorem for Feller semigroups in terms of boundary value problems (Theorem 4.13), generalizing the work of Bony-Courrège-Priouret [1], Sato-Ueno [14] and Taira [16] to the degenerate case. To do that, we consider the Dirichlet problem for differential operators which satisfy condition $(\mathrm{H})$ in the framework of Hölder spaces, following Oleĭnik-Radkevich [13] and Taira [17].

In Section 5 we prove an existence theorem for degenerate elliptic boundary value problems in the framework of Hölder spaces (Theorem 5.1) which plays an important role in the proof of Theorems 1.1.

Finally, Sections 6 and 7 are devoted to the proof of Theorem 1.1 and Theorem 1.2, respectively.

2. Theory of Feller semigroups. This section provides a brief description of the basic definitions and results about a class of semigroups 
associated with Markov processes. The semigroup approach to Markov processes can be traced back to the work of Kolmogorov [10]. It was substantially developed in the early 1950s, with Feller [6], [7] doing the pioneering work. Our presentation follows Dynkin [5], Lamperti [12] and Taira [16].

2.1. Markov transition functions and Feller semigroups. First we give the precise definition of a transition function which is adapted to our analysis. Let $(K, \varrho)$ be a locally compact, separable metric space and $\mathcal{B}$ the $\sigma$-algebra of all Borel sets in $K$. A function $p_{t}(x, E)$, defined for all $t \geq 0$, $x \in K$ and $E \in \mathcal{B}$, is called a (temporally homogeneous) Markov transition function on $K$ if it satisfies the following four conditions:

(a) $p_{t}(x, \cdot)$ is a non-negative measure on $\mathcal{B}$ and $p_{t}(x, K) \leq 1$ for each $t \geq 0$ and $x \in K$.

(b) $p_{t}(\cdot, E)$ is a Borel measurable function for each $t \geq 0$ and $E \in \mathcal{B}$.

(c) $p_{0}(x,\{x\})=1$ for each $x \in K$.

(d) (The Chapman-Kolmogorov equation) For any $t, s \geq 0, x \in K$ and $E \in \mathcal{B}$, we have

$$
p_{t+s}(x, E)=\int_{K} p_{t}(x, d y) p_{s}(y, E) .
$$

We add a point $\partial$ to $K$ as the point at infinity if $K$ is not compact, and as an isolated point if $K$ is compact; so the space $K_{\partial}=K \cup\{\partial\}$ is compact.

Let $C(K)$ be the space of real-valued, bounded continuous functions on $K$. It is a Banach space with the supremum norm

$$
\|f\|_{\infty}=\sup _{x \in K}|f(x)| .
$$

We say that a function $f$ in $C(K)$ converges to zero as $x \rightarrow \partial$ if, for each $\varepsilon>0$, there exists a compact subset $E$ of $K$ such that $|f(x)|<\varepsilon$ for $x \in K \backslash E$; we write $\lim _{x \rightarrow \partial} f(x)=0$. We let

$$
C_{0}(K)=\left\{f \in C(K): \lim _{x \rightarrow \partial} f(x)=0\right\} .
$$

Note that $C_{0}(K)$ may be identified with $C(K)$ if $K$ is compact.

If we introduce a useful convention that

- any real-valued function $f$ on $K$ is extended to the space $K_{\partial}=K \cup\{\partial\}$ by setting $f(\partial)=0$,

then

$$
C_{0}(K)=\left\{f \in C\left(K_{\partial}\right): f(\partial)=0\right\} .
$$

Furthermore we can extend a Markov transition function $p_{t}(x, \cdot)$ on $K$ to a Markov transition function $p_{t}^{\prime}(x, \cdot)$ on $K_{\partial}$ as follows: 


$$
\left\{\begin{array}{l}
p_{t}^{\prime}(x, E)=p_{t}(x, E), \quad x \in K, E \in \mathcal{B} \\
p_{t}^{\prime}(x,\{\partial\})=1-p_{t}(x, K), \quad x \in K \\
p_{t}^{\prime}(\partial, K)=0, \quad p_{t}^{\prime}(\partial,\{\partial\})=1
\end{array}\right.
$$

Intuitively, this means that a Markovian particle moves in $K$ until it "dies" when reaching the point $\partial$; hence $\partial$ is called the terminal point.

Now we introduce some conditions on the measures $p_{t}(x, \cdot)$ related to continuity in $x \in K$, for fixed $t \geq 0$.

A Markov transition function $p_{t}(x, \cdot)$ is called a Feller function if the function

$$
T_{t} f(x)=\int_{K} p_{t}(x, d y) f(y)
$$

is continuous in $x \in K$ whenever $f$ is in $C(K)$. We say that $p_{t}(x, \cdot)$ is a $C_{0}$-function if

$$
f \in C_{0}(K) \Rightarrow T_{t} f \in C_{0}(K) .
$$

A Markov transition function $p_{t}(x, \cdot)$ on $K$ is said to be uniformly stochastically continuous on $K$ if for each $\varepsilon>0$ and each compact $E \subset K$, we have

$$
\lim _{t \downarrow 0} \sup _{x \in E}\left[1-p_{t}\left(x, U_{\varepsilon}(x)\right)\right]=0,
$$

where $U_{\varepsilon}(x)=\{y \in K: \varrho(x, y)<\varepsilon\}$ is the $\varepsilon$-neighborhood of $x$.

Then we have the following (see [16, Theorem 9.2.3]):

Theorem 2.1. Let $p_{t}(x, \cdot)$ be a $C_{0}$-transition function on $K$. Then the operators $\left\{T_{t}\right\}_{t \geq 0}$ defined by

$$
T_{t} f(x)=\int_{K} p_{t}(x, d y) f(y), \quad f \in C_{0}(K),
$$

is strongly continuous in $t$ on $C_{0}(K)$ if and only if $p_{t}(x, \cdot)$ is uniformly stochastically continuous on $K$ and satisfies

$$
\lim _{x \rightarrow \partial} \sup _{0 \leq t \leq s} p_{t}(x, E)=0
$$

for each $s>0$ and each compact $E \subset K$.

A family $\left\{T_{t}\right\}_{t \geq 0}$ of bounded linear operators acting on $C_{0}(K)$ is called a Feller semigroup on $K$ if it satisfies the following three conditions:

(i) $T_{t+s}=T_{t} T_{s}, t, s \geq 0 ; T_{0}=I$.

(ii) The family $\left\{T_{t}\right\}$ is strongly continuous in $t$ for $t \geq 0$ :

$$
\lim _{s \downarrow 0}\left\|T_{t+s} f-T_{t} f\right\|_{\infty}=0, \quad f \in C_{0}(K) .
$$

(iii) The family $\left\{T_{t}\right\}$ is non-negative and contractive on $C_{0}(K)$ :

$$
f \in C_{0}(K), 0 \leq f(x) \leq 1 \text { on } K \Rightarrow 0 \leq T_{t} f(x) \leq 1 \text { on } K .
$$


The next theorem gives a characterization of Feller semigroups in terms of Markov transition functions (see [16, Theorem 9.2.6]):

TheOrem 2.2. If $p_{t}(x, \cdot)$ is a uniformly stochastically continuous $C_{0}$ transition function on $K$ and satisfies condition $(\mathrm{L})$, then the associated operators $\left\{T_{t}\right\}_{t \geq 0}$ form a Feller semigroup on $K$. Conversely, if $\left\{T_{t}\right\}_{t \geq 0}$ is a Feller semigroup on $K$, then there exists a uniformly stochastically continuous $C_{0}$-transition $p_{t}(x, \cdot)$ on $K$, satisfying condition $(\mathrm{L})$, such that formula (2.1) holds.

2.2. Generation theorems for Feller semigroups. If $\left\{T_{t}\right\}_{t \geq 0}$ is a Feller semigroup on $K$, we define its infinitesimal generator $\mathfrak{A}$ from $C_{0}(K)$ into itself defined by the formula

$$
\mathfrak{A} u=\lim _{t \downarrow 0} \frac{T_{t} u-u}{t}, \quad u \in \mathcal{D}(\mathfrak{A}),
$$

where

$$
\mathcal{D}(\mathfrak{A})=\left\{u \in C_{0}(K): \text { the limit }(2.2) \text { exists in } C_{0}(K)\right\} .
$$

The next theorem is a version of the Hille-Yosida theorem adapted to the present context (see [16, Theorem 9.3.1 and Corollary 9.3.2]):

Theorem 2.3. (i) Let $\left\{T_{t}\right\}_{t \geq 0}$ be a Feller semigroup on $K$ and $\mathfrak{A}$ its infinitesimal generator. Then we have the following:

(a) The domain $\mathcal{D}(\mathfrak{A})$ is dense in the space $C_{0}(K)$.

(b) For each $\alpha>0$, the equation $(\alpha I-\mathfrak{A}) u=f$ has a unique solution $u$ in $\mathcal{D}(\mathfrak{A})$ for any $f \in C_{0}(K)$. Hence, for each $\alpha>0$, the Green operator $(\alpha I-\mathfrak{A})^{-1}: C_{0}(K) \rightarrow C_{0}(K)$ can be defined by the formula

$$
u=(\alpha I-\mathfrak{A})^{-1} f, \quad f \in C_{0}(K) .
$$

(c) For each $\alpha>0$, the operator $(\alpha I-\mathfrak{A})^{-1}$ is non-negative on $C_{0}(K)$ :

$$
f \in C_{0}(K), f(x) \geq 0 \text { on } K \Rightarrow(\alpha I-\mathfrak{A})^{-1} f(x) \geq 0 \text { on } K .
$$

(d) For each $\alpha>0$, the operator $(\alpha I-\mathfrak{A})^{-1}$ is bounded on $C_{0}(K)$ with norm

$$
\left\|(\alpha I-\mathfrak{A})^{-1}\right\| \leq 1 / \alpha .
$$

(ii) Conversely, if $\mathfrak{A}$ is a linear operator from $C_{0}(K)$ into itself satisfying condition (a) and if there is a constant $\alpha_{0} \geq 0$ such that, for all $\alpha>\alpha_{0}$, conditions (b) through (d) are satisfied, then $\mathfrak{A}$ is the infinitesimal generator of some Feller semigroup $\left\{T_{t}\right\}_{t \geq 0}$ on $K$.

COROLlary 2.4. Let $K$ be a compact metric space and let $\mathfrak{A}$ be the infinitesimal generator of a Feller semigroup on $K$. Assume that the constant 
function 1 belongs to $\mathcal{D}(\mathfrak{A})$ and that for some constant $c$ we have

$$
\mathfrak{A} 1(x) \leq-c \quad \text { on } K .
$$

Then the operator $\mathfrak{A}^{\prime}=\mathfrak{A}+c I$ is the infinitesimal generator of some Feller semigroup on $K$.

Although Theorem 2.3 tells us precisely when a linear operator $\mathfrak{A}$ is the infinitesimal generator of some Feller semigroup, it is usually difficult to verify conditions (b) through (d). So we give useful criteria in terms of the maximum principle (see [1, Théorème de Hille-Yosida-Ray]; [16, Theorem 9.3.3 and Corollary 9.3.4]):

TheOREM 2.5. Let $K$ be a compact metric space. Then we have the following assertions:

(i) Let $B$ be a linear operator from $C(K)=C_{0}(K)$ into itself, and assume that:

$(\alpha)$ The domain $\mathcal{D}(B)$ is dense in $C(K)$.

$(\beta)$ There exists an open and dense subset $K_{0}$ of $K$ such that if $u \in \mathcal{D}(B)$ takes a positive maximum at a point $x_{0}$ of $K_{0}$, then

$$
B u\left(x_{0}\right) \leq 0 .
$$

Then the operator $B$ is closable in $C(K)$.

(ii) Let $B$ be as in part (i), and further assume that

$\left(\beta^{\prime}\right)$ If $u \in \mathcal{D}(B)$ takes a positive maximum at a point $x_{0}^{\prime}$ of $K$, then

$$
B u\left(x_{0}^{\prime}\right) \leq 0 .
$$

$(\gamma)$ For some $\alpha_{0} \geq 0$, the range $\mathcal{R}\left(\alpha_{0} I-B\right)$ is dense in $C(K)$.

Then the minimal closed extension $\bar{B}$ of $B$ is the infinitesimal generator of some Feller semigroup on $K$.

3. Theory of pseudo-differential operators. In this section we present a brief description of the basic concepts and results of the Hölder space theory of pseudo-differential operators which will be used in subsequent sections. In particular we recall an existence and uniqueness theorem for a class of pseudo-differential operators which enters naturally in the construction of Feller semigroups. For detailed studies of pseudo-differential operators, the reader is referred to Chazarain-Piriou [4], Hörmander [9], Kumano-go [11] and Taylor [20].

3.1. Function spaces. Let $\Omega$ be an open subset of $\mathbb{R}^{n}$. If $m$ is a nonnegative integer, we let $H^{m, \infty}(\Omega)$ be the space of equivalence classes of functions $u \in L^{\infty}(\Omega)$ all of whose derivatives $\partial^{\alpha} u,|\alpha| \leq m$, in the sense of 
distributions are in $L^{\infty}(\Omega)$. It is a Banach space with the norm

$$
\|u\|_{m, \infty}=\sum_{|\alpha| \leq m}\left\|\partial^{\alpha} u\right\|_{\infty}=\sum_{|\alpha| \leq m} \operatorname{ess} \sup _{x \in \Omega}\left|\partial^{\alpha} u(x)\right| .
$$

Here and in the following we use the shorthand

$$
\partial_{j}=\frac{\partial}{\partial x_{j}}, \quad 1 \leq j \leq n, \quad \partial^{\alpha}=\partial_{1}^{\alpha_{1}} \partial_{2}^{\alpha_{2}} \ldots \partial_{n}^{\alpha_{n}}, \quad \alpha=\left(\alpha_{1}, \alpha_{2}, \ldots, \alpha_{n}\right),
$$

for derivatives on $\mathbb{R}^{n}$.

Now let $\Omega$ be a bounded open subset of $\mathbb{R}^{n}$ and $0<\theta<1$. If $m$ is a non-negative integer, we let $C^{m+\theta}(\bar{\Omega})$ be the space of functions in $C^{m}(\bar{\Omega})$ all of whose $m$ th order derivatives are Hölder continuous with exponent $\theta$ on $\bar{\Omega}$. It is a Banach space with the norm

$$
\begin{aligned}
\|u\|_{C^{m+\theta}(\bar{\Omega})} & =\|u\|_{C^{m}(\bar{\Omega})}+\max _{|\alpha|=m}\left[\partial^{\alpha} u\right]_{\theta ; \bar{\Omega}} \\
& =\sum_{|\alpha| \leq m} \max _{x \in \bar{\Omega}}\left|\partial^{\alpha} u(x)\right|+\max _{|\alpha|=m} \sup _{\substack{x, y \in \bar{\Omega} \\
x \neq y}} \frac{\left|\partial^{\alpha} u(x)-\partial^{\alpha} u(y)\right|}{|x-y|^{\theta}} .
\end{aligned}
$$

If $M$ is an $n$-dimensional compact smooth manifold without boundary, then the spaces $H^{m, \infty}(M)$ and $C^{m+\theta}(M)$ are defined respectively to be locally the spaces $H^{m, \infty}\left(\mathbb{R}^{n}\right)$ and $C^{m+\theta}\left(\mathbb{R}^{n}\right)$, upon using local coordinate systems flattening out $M$, together with a partition of unity. The norms of the spaces $H^{m, \infty}(M)$ and $C^{m+\theta}(M)$ will be denoted by $\|\cdot\|_{m, \infty}$ and $\|\cdot\|_{C^{m+\theta}(M)}$, respectively.

We recall the following results (see Triebel [21]):

(I) If $k$ is a positive integer, then

$$
H^{k, \infty}(M)=\left\{\varphi \in C^{k-1}(M): \max _{|\alpha| \leq k-1} \sup _{\substack{x, y \in M \\ x \neq y}} \frac{\left|\partial^{\alpha} \varphi(x)-\partial^{\alpha} \varphi(y)\right|}{|x-y|}<\infty\right\},
$$

where $|x-y|$ is the geodesic distance between $x$ and $y$ with respect to the Riemannian metric of $M$.

(II) The space $C^{k+\theta}(M)$ is a real interpolation space between $H^{k, \infty}(M)$ and $H^{k+1, \infty}(M)$. More precisely we have

$$
\begin{aligned}
C^{k+\theta}(M) & =\left(H^{k, \infty}(M), H^{k+1, \infty}(M)\right)_{\theta, \infty} \\
& =\left\{u \in H^{k, \infty}(M): \sup _{t>0} K(t, u) / t^{\theta}<\infty\right\},
\end{aligned}
$$

where

$$
\begin{aligned}
& K(t, u)=\inf \left\{\left\|u_{0}\right\|_{k, \infty}+t\left\|u_{1}\right\|_{k+1, \infty}: u=u_{0}+u_{1},\right. \\
&\left.u_{0} \in H^{k, \infty}(M), u_{1} \in H^{k+1, \infty}(M)\right\} .
\end{aligned}
$$


3.2. Pseudo-differential operators. Let $\Omega$ be an open subset of $\mathbb{R}^{n}$. If $m \in \mathbb{R}$ and $0 \leq \delta<\varrho \leq 1$, we let $S_{\varrho, \delta}^{m}\left(\Omega \times \mathbb{R}^{N}\right)$ be the set of all functions $a \in C^{\infty}\left(\Omega \times \mathbb{R}^{N}\right)$ with the property that, for any compact $K \subset \Omega$ and multi-indices $\alpha, \beta$, there exists a constant $C_{K, \alpha, \beta}>0$ such that for all $x \in K$ and $\theta \in \mathbb{R}^{N}$ we have

$$
\left|\partial_{\theta}^{\alpha} \partial_{x}^{\beta} a(x, \theta)\right| \leq C_{K, \alpha, \beta}(1+|\theta|)^{m-\varrho|\alpha|+\delta|\beta|} .
$$

The elements of $S_{\varrho, \delta}^{m}\left(\Omega \times \mathbb{R}^{N}\right)$ are called symbols of order $m$.

We set

$$
S^{-\infty}\left(\Omega \times \mathbb{R}^{N}\right)=\bigcap_{m \in \mathbb{R}} S_{\varrho, \delta}^{m}\left(\Omega \times \mathbb{R}^{N}\right) .
$$

If $a_{j} \in S_{\varrho, \delta}^{m_{j}}\left(\Omega \times \mathbb{R}^{N}\right)$ is a sequence of symbols of decreasing orders, then there exists a symbol $a \in S_{\varrho, \delta}^{m_{0}}\left(\Omega \times \mathbb{R}^{N}\right)$, unique modulo $S^{-\infty}\left(\Omega \times \mathbb{R}^{N}\right)$, such that for all $k>0$ we have

$$
a-\sum_{j=0}^{k-1} a_{j} \in S_{\varrho, \delta}^{m_{k}}\left(\Omega \times \mathbb{R}^{N}\right) .
$$

In this case, we write $a \sim \sum_{j=0}^{\infty} a_{j}$.

A symbol $a(x, \theta) \in S_{1,0}^{m}\left(\Omega \times \mathbb{R}^{N}\right)$ is said to be classical if there exist smooth functions $a_{j}(x, \theta)$, positively homogeneous of degree $m-j$ in $\theta$ for $|\theta| \geq 1$, such that $a \sim \sum_{j=0}^{\infty} a_{j}$. We let $S_{\mathrm{cl}}^{m}\left(\Omega \times \mathbb{R}^{N}\right)$ be the set of all classical symbols of order $m$.

Let $\Omega$ be an open subset of $\mathbb{R}^{n}$ and $m \in \mathbb{R}$. A pseudo-differential operator of order $m$ on $\Omega$ is a Fourier integral operator of the form

$$
A u(x)=\iint_{\Omega \times \mathbb{R}^{n}} e^{i(x-y) \cdot \xi} a(x, y, \xi) u(y) d y d \xi, \quad u \in C_{0}^{\infty}(\Omega),
$$

with some $a \in S_{\varrho, \delta}^{m}\left(\Omega \times \Omega \times \mathbb{R}^{n}\right)$. Here the integral is taken in the sense of oscillatory integrals. We let $L_{\varrho, \delta}^{m}(\Omega)$ be the set of all pseudo-differential operators of order $m$ on $\Omega$, and set

$$
L^{-\infty}(\Omega)=\bigcap_{m \in \mathbb{R}} L_{\varrho, \delta}^{m}(\Omega) .
$$

If $A \in L_{\varrho, \delta}^{m}(\Omega)$, one can choose a properly supported operator $A_{0} \in$ $L_{\varrho, \delta}^{m}(\Omega)$ such that $A-A_{0} \in L^{-\infty}(\Omega)$, and define $\sigma(A)$ to be the equivalence class of the complete symbol of $A_{0}$ in $S_{\varrho, \delta}^{m}\left(\Omega \times \mathbb{R}^{n}\right) / S^{-\infty}\left(\Omega \times \mathbb{R}^{n}\right)$. The equivalence class $\sigma(A)$ does not depend on the operator $A_{0}$ chosen, and is called the complete symbol of $A$. We often identify the complete symbol $\sigma(A)$ with a representative in the class $S_{\varrho, \delta}^{m}\left(\Omega \times \mathbb{R}^{n}\right)$ for notational convenience, and call any member of $\sigma(A)$ a complete symbol of $A$. 
A pseudo-differential operator $A \in L_{1,0}^{m}(\Omega)$ is said to be classical if its complete symbol $\sigma(A)$ has a representative in $S_{\mathrm{cl}}^{m}\left(\Omega \times \mathbb{R}^{n}\right)$. We let $L_{\mathrm{cl}}^{m}(\Omega)$ be the set of all classical pseudo-differential operators of order $m$ on $\Omega$.

Now we define the concept of a pseudo-differential operator on a manifold, and transfer all the machinery of pseudo-differential operators to manifolds.

Let $M$ be an $n$-dimensional, compact smooth manifold without boundary and $1-\varrho \leq \delta<\varrho \leq 1$. A continuous linear operator $A: C^{\infty}(M) \rightarrow C^{\infty}(M)$ is called a pseudo-differential operator of order $m \in \mathbb{R}$ if it satisfies the following two conditions:

(i) The distribution kernel of $A$ is smooth off the diagonal $\Delta_{M}=\{(x, x)$ : $x \in M\}$ in $M \times M$.

(ii) For any chart $(U, \chi)$ on $M$, the mapping

$$
A_{\chi}: C_{0}^{\infty}(\chi(U)) \rightarrow C^{\infty}(\chi(U)), \quad u \mapsto A(u \circ \chi) \circ \chi^{-1},
$$

belongs to the class $L_{\varrho, \delta}^{m}(\chi(U))$.

We let $L_{\varrho, \delta}^{m}(M)$ be the set of all pseudo-differential operators of order $m$ on $M$, and set

$$
L^{-\infty}(M)=\bigcap_{m \in \mathbb{R}} L_{\varrho, \delta}^{m}(M)
$$

Some results about pseudo-differential operators on $\mathbb{R}^{n}$ are also true for pseudo-differential operators on $M$, since pseudo-differential operators on $M$ are defined to be locally pseudo-differential operators on $\mathbb{R}^{n}$.

For example we have the following results:

(1) A pseudo-differential operator $A$ extends to a continuous linear operator $A: \mathcal{D}^{\prime}(M) \rightarrow \mathcal{D}^{\prime}(M)$.

(2) $\operatorname{sing} \operatorname{supp} A u \subset \operatorname{sing} \operatorname{supp} u, u \in \mathcal{D}^{\prime}(M)$.

(3) A continuous linear operator $A: C^{\infty}(M) \rightarrow \mathcal{D}^{\prime}(M)$ is a regularizer if and only if it is in $L^{-\infty}(M)$.

(4) The class $L_{\varrho, \delta}^{m}(M), 1-\varrho \leq \delta<\varrho \leq 1$, is stable under the operations of composition of operators and taking the transpose or adjoint of an operator.

(5) A pseudo-differential operator $A \in L_{1,0}^{m}(M)$ extends to a continuous linear operator $A: C^{k+\theta}(M) \rightarrow C^{k-m+\theta}(M)$ for any integer $k \geq m$.

A pseudo-differential operator $A \in L_{1,0}^{m}(M)$ is said to be classical if, for any chart $(U, \chi)$ on $M$, the mapping $A_{\chi}: C_{0}^{\infty}(\chi(U)) \rightarrow C^{\infty}(\chi(U))$ belongs to the class $L_{\mathrm{cl}}^{m}(\chi(U))$. We let $L_{\mathrm{cl}}^{m}(M)$ be the set of all classical pseudo-differential operators of order $m$ on $M$. We observe that

$$
L^{-\infty}(M)=\bigcap_{m \in \mathbb{R}} L_{\mathrm{cl}}^{m}(M) .
$$


3.3. Unique solvability theorem for pseudo-differential operators. The next result will play an essential role in the construction of Feller semigroups in Sections 5-7 (see [2, Théorème 4.5], [19, Theorem 2.1]):

THEOREM 3.1. Let $T$ be a classical pseudo-differential operator of second order on a compact smooth manifold $M$ without boundary such that

$$
T=P+S,
$$

where:

(a) The operator $P$ is a second-order degenerate elliptic differential operator on $M$ with non-positive principal symbol, and $P 1 \leq 0$ on $M$.

(b) The operator $S$ is a classical pseudo-differential operator of order $2-\kappa, \kappa>0$, on $M$ and its distribution kernel $s(x, y)$ is non-negative off the diagonal $\Delta_{M}=\{(x, x): x \in M\}$ in $M \times M$.

(c) $T 1=P 1+S 1 \leq 0$ on $M$.

Then, for each integer $k \geq 1$, there exists a constant $\lambda=\lambda(k)>0$ such that for any $f \in C^{k+\theta}(M)$ one can find a function $\varphi \in C^{k+\theta}(M)$ satisfying the equation

$$
(T-\lambda) \varphi=f \quad \text { on } M
$$

and the estimate

$$
\|\varphi\|_{C^{k+\theta}(M)} \leq C\|f\|_{C^{k+\theta}(M)} .
$$

Here $C>0$ is a constant independent of $f$.

4. Feller semigroups and boundary value problems. The purpose of this section is to give a general existence theorem for Feller semigroups in terms of boundary value problems, generalizing the work of Bony-CourrègePriouret [1], Sato-Ueno [14] and Taira [16] to the degenerate case. To do that, we consider a differential operator $A$ which satisfies condition $(\mathrm{H})$, and study the Dirichlet problem in the framework of Hölder spaces. For more thorough treatments of this subject, the reader is referred to OleŭnikRadkevich [13] and Taira [17].

4.1. The Dirichlet problem. First we consider the following Dirichlet problem: Given functions $f(x)$ and $\varphi\left(x^{\prime}\right)$ defined in $D$ and on $\Sigma_{2}$, respectively, find a function $u(x)$ in $D$ such that

$$
\begin{cases}(\alpha-A) u=f & \text { in } D \\ \left.u\right|_{\Sigma_{2}}=\varphi & \text { on } \Sigma_{2}\end{cases}
$$

where $\alpha$ is a positive parameter.

The next result states an existence and uniqueness theorem for problem (D) in the framework of Hölder spaces (see [13, Theorem 1.8.2], [17, Theorem 2]): 
TheOREM 4.1. Assume that hypothesis $(\mathrm{H})$ is satisfied. Then for each integer $m \geq 2$ one can find a constant $\alpha=\alpha(m)>0$ such that for any $f \in C^{2 m+2+2 \theta}(\bar{D})$ and any $\varphi \in C^{2 m+4+2 \theta}\left(\Sigma_{2}\right)$ with $0<\theta<1$ there exists a unique solution $u \in C^{m+\theta}(\bar{D})$ of problem $(\mathrm{D})$.

Now, if we take $m=2$ in Theorem 4.1 and let

$$
\alpha \geq \alpha(2),
$$

then we can introduce two linear operators

$$
G_{\alpha}^{0}: C^{6+2 \theta}(\bar{D}) \rightarrow C^{2+\theta}(\bar{D}), \quad H_{\alpha}: C^{8+2 \theta}\left(\Sigma_{2}\right) \rightarrow C^{2+\theta}(\bar{D})
$$

as follows.

(a) For any $f \in C^{6+2 \theta}(\bar{D})$, the function $G_{\alpha}^{0} f \in C^{2+\theta}(\bar{D})$ is the unique solution of the problem

$$
\begin{cases}(\alpha-A) G_{\alpha}^{0} f=f & \text { in } D \\ \left.G_{\alpha}^{0} f\right|_{\Sigma_{2}}=0 & \text { on } \Sigma_{2} .\end{cases}
$$

(b) For any $\varphi \in C^{8+2 \theta}\left(\Sigma_{2}\right)$, the function $H_{\alpha} \varphi \in C^{2+\theta}(\bar{D})$ is the unique solution of the problem

$$
\begin{cases}(\alpha-A) H_{\alpha} \varphi=0 & \text { in } D \\ \left.H_{\alpha} \varphi\right|_{\Sigma_{2}}=\varphi & \text { on } \Sigma_{2} .\end{cases}
$$

The operator $G_{\alpha}^{0}$ is called the Green operator, and $H_{\alpha}$ is called the harmonic operator, respectively.

Then we have the following results (see [19, Lemmas 4.2 and 4.3]):

LEMmA 4.2. The operator $G_{\alpha}^{0}$, considered from $C(\bar{D})$ into itself, is nonnegative and continuous with norm

$$
\left\|G_{\alpha}^{0}\right\|=\left\|G_{\alpha}^{0} 1\right\|_{\infty}=\max _{x \in \bar{D}} G_{\alpha}^{0} 1(x) .
$$

Lemma 4.3. The operator $H_{\alpha}$, considered from $C\left(\Sigma_{2}\right)$ into $C(\bar{D})$, is non-negative and continuous with norm

$$
\left\|H_{\alpha}\right\|=\left\|H_{\alpha} 1\right\|_{\infty}=\max _{x \in \bar{D}} H_{\alpha} 1(x) .
$$

More precisely we have the following (see [19, Theorem 4.4]):

Theorem 4.4.

(i) (a) The operator $G_{\alpha}^{0}$ can be uniquely extended to a non-negative, bounded linear operator on $C(\bar{D})$ into itself, denoted again by $G_{\alpha}^{0}$, with norm

$$
\left\|G_{\alpha}^{0}\right\|=\left\|G_{\alpha}^{0} 1\right\|_{\infty} \leq 1 / \alpha .
$$

(b) For any $f \in C(\bar{D})$, we have $\left.G_{\alpha}^{0} f\right|_{\Sigma_{2}}=0$ on $\Sigma_{2}$. 
(c) For all $\alpha, \beta$, the resolvent equation holds:

$$
G_{\alpha}^{0} f-G_{\beta}^{0} f+(\alpha-\beta) G_{\alpha}^{0} G_{\beta}^{0} f=0, \quad f \in C(\bar{D}) .
$$

(d) For any $f \in C(\bar{D})$, we have

$$
\lim _{\alpha \rightarrow+\infty} \alpha G_{\alpha}^{0} f(x)=f(x), \quad x \in D .
$$

Furthermore, if $\left.f\right|_{\Sigma_{2}}=0$, then this convergence is uniform in $x \in \bar{D}$, that is,

$$
\lim _{\alpha \rightarrow+\infty} \alpha G_{\alpha}^{0} f=f \quad \text { in } C(\bar{D}) .
$$

(ii) (a') The operator $H_{\alpha}$ can be uniquely extended to a non-negative, bounded linear operator on $C\left(\Sigma_{2}\right)$ into $C(\bar{D})$, denoted again by $H_{\alpha}$, with norm $\left\|H_{\alpha}\right\|=1$.

(b') For any $\varphi \in C\left(\Sigma_{2}\right)$, we have $\left.H_{\alpha} \varphi\right|_{\Sigma_{2}}=\varphi$ on $\Sigma_{2}$.

$\left(\mathrm{c}^{\prime}\right)$ For all $\alpha, \beta$, we have

$$
H_{\alpha} \varphi-H_{\beta} \varphi+(\alpha-\beta) G_{\alpha}^{0} H_{\beta} \varphi=0, \quad \varphi \in C\left(\Sigma_{2}\right) .
$$

4.2. Existence theorem for Feller semigroups. It is known (see [1], [14], [16], [22]) that the infinitesimal generator $\mathfrak{A}$ of a Feller semigroup $\left\{T_{t}\right\}_{t \geq 0}$ is described analytically by a second-order differential operator $A$ and a Wentzell boundary condition $L$.

We are interested in the following:

Problem. Conversely, given analytic data $(A, L)$, can we construct a Feller semigroup $\left\{T_{t}\right\}_{t \geq 0}$ on $\bar{D}$ whose infinitesimal generator $\mathfrak{A}$ is characterized by $(A, L)$ ?

Now we consider the following boundary value problem $(*)$ in the framework of spaces of continuous functions:

$$
\begin{cases}(\alpha-A) u=f & \text { in } D \\ L u=\varphi & \text { on } \Sigma_{2}\end{cases}
$$

To this end, we introduce three operators associated with problem $(*)$.

(I) First we introduce a linear operator $A: C(\bar{D}) \rightarrow C(\bar{D})$ by

$$
A u=\sum_{i, j=1}^{N} a^{i j}(x) \frac{\partial^{2} u}{\partial x_{i} \partial x_{j}}+\sum_{i=1}^{N} b^{i}(x) \frac{\partial u}{\partial x_{i}}+c(x) u, \quad u \in \mathcal{D}(A),
$$

where $\mathcal{D}(A)=C^{2}(\bar{D})$.

Then we have the following (see [19, Lemma 4.5]):

Lemma 4.5. The operator A has minimal closed extension $\bar{A}$ in the space $C(\bar{D})$. 
The extended operators $G_{\alpha}^{0}: C(\bar{D}) \rightarrow C(\bar{D})$ and $H_{\alpha}: C\left(\Sigma_{2}\right) \rightarrow C(\bar{D})$ still satisfy formulas (4.1) and (4.2) respectively in the following sense (see [19, Lemma 4.7]):

Lemma 4.6. (i) For any $f \in C(\bar{D})$, we have

$$
\left\{\begin{array}{l}
G_{\alpha}^{0} f \in \mathcal{D}(\bar{A}), \\
(\alpha I-\bar{A}) G_{\alpha}^{0} f=f \quad \text { in } D .
\end{array}\right.
$$

(ii) For any $\varphi \in C\left(\Sigma_{2}\right)$, we have

$$
\left\{\begin{array}{l}
H_{\alpha} \varphi \in \mathcal{D}(\bar{A}) \\
(\alpha I-\bar{A}) H_{\alpha} \varphi=0 \quad \text { in } D .
\end{array}\right.
$$

Here $\mathcal{D}(\bar{A})$ is the domain of the closed extension $\bar{A}$.

Furthermore we have the following (see [19, Corollary 4.8]):

Corollary 4.7. Every function $u \in \mathcal{D}(\bar{A})$ can be written in the following form:

$$
u=G_{\alpha}^{0}((\alpha I-\bar{A}) u)+H_{\alpha}\left(\left.u\right|_{\Sigma_{2}}\right) .
$$

(II) Secondly we introduce a linear operator

$$
L G_{\alpha}^{0}: C(\bar{D}) \rightarrow C\left(\Sigma_{2}\right)
$$

by

$$
L G_{\alpha}^{0} f=L\left(G_{\alpha}^{0} f\right), \quad f \in \mathcal{D}\left(L G_{\alpha}^{0}\right)=C^{6+2 \theta}(\bar{D}) .
$$

Then we have the following (see [19, Lemma 4.9]):

LEMMA 4.8. The operator $L G_{\alpha}^{0}$ can be uniquely extended to a non-negative, bounded linear operator $\overline{L G_{\alpha}^{0}}: C(\bar{D}) \rightarrow C\left(\Sigma_{2}\right)$.

The next lemma states a fundamental relationship between the operators $\overline{L G_{\alpha}^{0}}$ and $\overline{L G_{\beta}^{0}}$ (see [19, Lemma 4.10]):

Lemma 4.9. For any $f \in C(\bar{D})$, we have

$$
\overline{L G_{\alpha}^{0}} f-\overline{L G_{\beta}^{0}} f+(\alpha-\beta) \overline{L G_{\alpha}^{0}} G_{\beta}^{0} f=0 .
$$

(III) Finally we introduce a linear operator

$$
L H_{\alpha}: C\left(\Sigma_{2}\right) \rightarrow C\left(\Sigma_{2}\right)
$$

by

$$
L H_{\alpha} \psi=L\left(H_{\alpha} \psi\right), \quad \psi \in \mathcal{D}\left(L H_{\alpha}\right)=C^{8+2 \theta}\left(\Sigma_{2}\right) .
$$

Then we have the following:

LEMma 4.10. The operator $L H_{\alpha}$ has a minimal closed extension $\overline{L H_{\alpha}}$ in the space $C\left(\Sigma_{2}\right)$. 
Proof. We apply Theorem 2.5(i) with $K:=K_{0}=\Sigma_{2}$ to the operator $B:=L H_{\alpha}$. To do that, it suffices to show that the operator $L H_{\alpha}$ satisfies condition $(\beta)$ of the theorem.

The next claim is an essential step in the proof:

Claim 4.11. If $\left(x_{1}, \ldots, x_{N-1}\right)$ are local coordinates on $\Sigma_{2}$, then the function $(\partial / \partial \mathbf{n})\left(H_{\alpha} \varphi\right), \varphi \in C^{\infty}\left(\Sigma_{2}\right)$, is given by the formula

$$
\begin{aligned}
& \frac{\partial}{\partial \mathbf{n}}\left(H_{\alpha} \varphi\right) \\
& \quad=-\frac{1}{b\left(x^{\prime}\right)}\left(\sum_{i, j=1}^{N-1} a^{i j}\left(x^{\prime}\right) \frac{\partial^{2} \varphi}{\partial x_{i} \partial x_{j}}+\sum_{i=1}^{N-1} b^{i}\left(x^{\prime}\right) \frac{\partial \varphi}{\partial x_{i}}+\left(c\left(x^{\prime}\right)-\alpha\right) \varphi\right),
\end{aligned}
$$

where $b\left(x^{\prime}\right)$ is the Fichera function for the operator $A$.

Proof. Let $x_{0}^{\prime} \in \Sigma_{2}$. We choose a local coordinate system $\left(x_{1}, \ldots, x_{N}\right)$

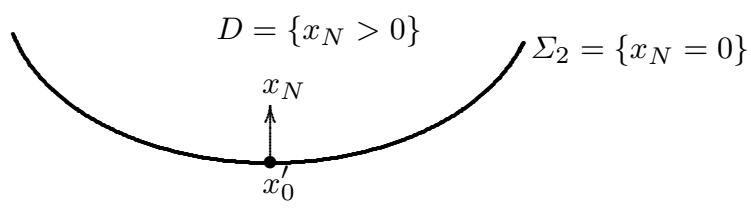

Fig. 4.1

in a neighborhood of $x_{0}^{\prime}$ such that (see Figure 4.1)

$$
x_{0}^{\prime}=0, \quad D=\left\{x_{N}>0\right\}, \quad \Sigma_{2}=\left\{x_{N}=0\right\},
$$

and assume that, in this coordinate system,

$$
\begin{aligned}
A u= & a^{N N}(x) \frac{\partial^{2} u}{\partial x_{N}^{2}}+b^{N}(x) \frac{\partial u}{\partial x_{N}} \\
& +\sum_{i, j=1}^{N-1} a^{i j}(x) \frac{\partial^{2} u}{\partial x_{i} \partial x_{j}}+\sum_{i=1}^{N-1} b^{i}(x) \frac{\partial u}{\partial x_{i}}+c(x) u .
\end{aligned}
$$

We remark that:

- $a^{N N}(0)=0$ and $b^{N}(0)=b(0)<0$, since $x_{0}^{\prime}=0 \in \Sigma_{2}$.

- $a^{i j} \in C^{\infty}\left(\mathbb{R}^{N}\right), a^{i j}(x)=a^{j i}(x)$ and

$$
\sum_{i, j=1}^{N} a^{i j}(x) \xi_{i} \xi_{j} \geq 0, \quad x \in \mathbb{R}^{N}, \xi \in \mathbb{R}^{N} .
$$

Since

$$
\begin{cases}(A-\alpha) H_{\alpha} \varphi=0 & \text { in } D \\ H_{\alpha} \varphi=\varphi & \text { on } \Sigma_{2}\end{cases}
$$

it follows that 


$$
\begin{aligned}
0= & \alpha \varphi(0)-\left(a^{N N}(0) \frac{\partial^{2}}{\partial x_{N}^{2}}\left(H_{\alpha} \varphi\right)(0)+b^{N}(0) \frac{\partial}{\partial x_{N}}\left(H_{\alpha} \varphi\right)(0)\right. \\
& \left.+\sum_{i, j=1}^{N-1} a^{i j}(0) \frac{\partial^{2} \varphi}{\partial x_{i} \partial x_{j}}(0)+\sum_{i=1}^{N-1} b^{i}(0) \frac{\partial \varphi}{\partial x_{i}}(0)+c(0) \varphi(0)\right) \\
= & \alpha \varphi(0)-\left(b^{N}(0) \frac{\partial}{\partial x_{N}}\left(H_{\alpha} \varphi\right)(0)+\sum_{i, j=1}^{N-1} a^{i j}(0) \frac{\partial^{2} \varphi}{\partial x_{i} \partial x_{j}}(0)\right. \\
& \left.+\sum_{i=1}^{N-1} b^{i}(0) \frac{\partial \varphi}{\partial x_{i}}(0)+c(0) \varphi(0)\right)
\end{aligned}
$$

This proves that

$$
\begin{aligned}
& \frac{\partial}{\partial \mathbf{n}}\left(H_{\alpha} \varphi\right)\left(x_{0}^{\prime}\right)=\frac{\partial}{\partial x_{N}}\left(H_{\alpha} \varphi\right)(0) \\
& =-\frac{1}{b^{N}(0)}\left(\sum_{i, j=1}^{N-1} a^{i j}(0) \frac{\partial^{2} \varphi}{\partial x_{i} \partial x_{j}}(0)+\sum_{i=1}^{N-1} b^{i}(0) \frac{\partial \varphi}{\partial x_{i}}(0)+(c(0)-\alpha) \varphi(0)\right) \\
& =-\frac{1}{b\left(x_{0}^{\prime}\right)} \\
& \quad \times\left(\sum_{i, j=1}^{N-1} a^{i j}\left(x_{0}^{\prime}\right) \frac{\partial^{2} \varphi}{\partial x_{i} \partial x_{j}}\left(x_{0}^{\prime}\right)+\sum_{i=1}^{N-1} b^{i}\left(x_{0}^{\prime}\right) \frac{\partial \varphi}{\partial x_{i}}\left(x_{0}^{\prime}\right)+\left(c\left(x_{0}^{\prime}\right)-\alpha\right) \varphi\left(x_{0}^{\prime}\right)\right) .
\end{aligned}
$$

The proof of Claim 4.11 is complete.

Now assume that a function $\psi$ in $\mathcal{D}\left(L H_{\alpha}\right)=C^{8+2 \theta}\left(\Sigma_{2}\right)$ takes its positive maximum at some point $x_{0}^{\prime}$ of $\Sigma_{2}$. Since $H_{\alpha} \psi$ is in $C^{2+\theta}(\bar{D})$ and satisfies

$$
\begin{cases}(A-\alpha) H_{\alpha} \psi=0 & \text { in } D, \\ \left.H_{\alpha} \psi\right|_{\Sigma_{2}}=\psi & \text { on } \Sigma_{2},\end{cases}
$$

applying [19, Theorem 7.1 (the weak maximum principle)] we find that $H_{\alpha} \psi$ takes its positive maximum $\psi\left(x_{0}^{\prime}\right)$ at $x_{0}^{\prime} \in \Sigma_{2}$.

Hence, by formula (4.9) with $\varphi:=\psi$,

$$
\begin{aligned}
L H_{\alpha} \psi\left(x_{0}^{\prime}\right)= & \sum_{i, j=1}^{N-1} \alpha^{i j}\left(x_{0}^{\prime}\right) \frac{\partial^{2} \psi}{\partial x_{i} \partial x_{j}}\left(x_{0}^{\prime}\right)+\left(\gamma\left(x_{0}^{\prime}\right)-\alpha \delta\left(x_{0}^{\prime}\right)\right) \psi\left(x_{0}^{\prime}\right) \\
& +\left(-\frac{\mu\left(x_{0}^{\prime}\right)}{b\left(x_{0}^{\prime}\right)}\right)\left[\sum_{i, j=1}^{N-1} a^{i j}\left(x_{0}^{\prime}\right) \frac{\partial^{2} \psi}{\partial x_{i} \partial x_{j}}\left(x_{0}^{\prime}\right)+\left(c\left(x_{0}^{\prime}\right)-\alpha\right) \psi\left(x_{0}^{\prime}\right)\right] \\
& +\int_{\Sigma_{2}} r\left(x_{0}^{\prime}, y^{\prime}\right)\left(\psi\left(y^{\prime}\right)-\psi\left(x_{0}^{\prime}\right)\right) d y^{\prime} \leq 0 .
\end{aligned}
$$

This verifies condition $(\beta)$ of Theorem 2.5. 
Therefore Lemma 4.10 follows from an application of Theorem 2.5.

The next lemma states a fundamental relationship between the operators $\overline{L H_{\alpha}}$ and $\overline{L H_{\beta}}$ (see [19, Lemma 4.13]):

Lemma 4.12. The domain $\mathcal{D}\left(\overline{L H_{\alpha}}\right)$ does not depend on $\alpha$; denote it by $\mathcal{D}$. Then

$$
\overline{L H_{\alpha}} \psi-\overline{L H_{\beta}} \psi+(\alpha-\beta) \overline{L G_{\alpha}^{0}} H_{\beta} \psi=0, \quad \psi \in \mathcal{D} .
$$

Now we can give a general existence theorem for Feller semigroups on $\Sigma_{2}$ in terms of the boundary value problem $(*)$. The next theorem asserts that $\overline{L H_{\alpha}}$ is the infinitesimal generator of some Feller semigroup on $\Sigma_{2}$ if and only if problem $(*)$ is solvable for sufficiently many $\varphi$ in $C\left(\Sigma_{2}\right)$ :

THEOREM 4.13. (i) If the operator $\overline{L H_{\alpha}}$ is the infinitesimal generator of a Feller semigroup on $\Sigma_{2}$, then, for each constant $\lambda>0$, the boundary value problem

$(* *)_{0}$

$$
\begin{cases}(\alpha-A) u=0 & \text { in } D \\ (\lambda-L) u=\varphi & \text { on } \Sigma_{2}\end{cases}
$$

has a solution $u \in C^{2+\theta}(\bar{D})$ for any $\varphi$ in some dense subset of $C\left(\Sigma_{2}\right)$.

(ii) Conversely, if, for some constant $\lambda \geq 0$, problem $(* *)_{0}$ has a solution $u \in C^{8+2 \theta}(\bar{D})$ for any $\varphi$ in some dense subset of $C\left(\Sigma_{2}\right)$, then $\overline{L H_{\alpha}}$ is the infinitesimal generator of some Feller semigroup on $\Sigma_{2}$.

Proof. (i) If $\overline{L H_{\alpha}}$ generates a Feller semigroup on $\Sigma_{2}$, then applying Theorem 2.3(i) with $K:=\Sigma_{2}$ to the operator $\mathfrak{A}:=\overline{L H_{\alpha}}$ we obtain, for each $\lambda>0$,

$$
\mathcal{R}\left(\lambda I-\overline{L H_{\alpha}}\right)=C\left(\Sigma_{2}\right) .
$$

This implies that $\mathcal{R}\left(\lambda I-L H_{\alpha}\right)$ is dense in $C\left(\Sigma_{2}\right)$ for each $\lambda>0$. If $\varphi \in$ $C\left(\Sigma_{2}\right)$ is in $\mathcal{R}\left(\lambda I-L H_{\alpha}\right)$, that is, $\varphi=\left(\lambda I-L H_{\alpha}\right) \psi$ with $\psi \in C^{8+2 \theta}\left(\Sigma_{2}\right)$, then $u=H_{\alpha} \psi \in C^{2+\theta}(\bar{D})$ is a solution of $(* *)_{0}$.

(ii) We apply Theorem 2.5(ii) with $K:=\Sigma_{2}$ to the operator $L H_{\alpha}$. To do that, it suffices to show that $L H_{\alpha}$ satisfies condition $(\gamma)$ of the theorem, since it satisfies condition $\left(\beta^{\prime}\right)$, as is shown in the proof of Lemma 4.10.

By the uniqueness theorem for problem (D) (Theorem 4.1), any function $u \in C^{8+2 \theta}(\bar{D})$ which satisfies the equation $(\alpha-A) u=0$ in $D$ can be written in the form

$$
u=H_{\alpha}\left(\left.u\right|_{\Sigma_{2}}\right),\left.\quad u\right|_{\Sigma_{2}} \in C^{8+2 \theta}\left(\Sigma_{2}\right)=\mathcal{D}\left(L H_{\alpha}\right) .
$$

Thus if there exists a solution $u \in C^{8+2 \theta}(\bar{D})$ of $(* *)_{0}$ for a function $\varphi \in$ $C\left(\Sigma_{2}\right)$, then

$$
\left(\lambda I-L H_{\alpha}\right)\left(\left.u\right|_{\Sigma_{2}}\right)=\varphi,
$$

and so $\varphi \in \mathcal{R}\left(\lambda I-L H_{\alpha}\right)$. Therefore, if, for some constant $\lambda \geq 0$, problem $(* *)_{0}$ has a solution $u \in C^{8+2 \theta}(\bar{D})$ for any $\varphi$ in some dense subset of $C\left(\Sigma_{2}\right)$, 
then $\mathcal{R}\left(\lambda I-L H_{\alpha}\right)$ is dense in $C\left(\Sigma_{2}\right)$. This verifies condition $(\gamma)$ of Theorem 2.5 with $\alpha_{0}:=\lambda$ and completes the proof.

We conclude this section by giving a precise meaning to the boundary conditions $L u$ for functions $u \in \mathcal{D}(\bar{A})$.

We let

$$
\mathcal{D}(L)=\left\{u \in \mathcal{D}(\bar{A}):\left.u\right|_{\Sigma_{2}} \in \mathcal{D}\right\},
$$

where $\mathcal{D}$ is the common domain of the operators $\overline{L H_{\alpha}}$. We remark that $\mathcal{D}(L)$ contains $C^{8+2 \theta}(\bar{D})$, since $C^{8+2 \theta}\left(\Sigma_{2}\right)=\mathcal{D}\left(L H_{\alpha}\right) \subset \mathcal{D}$. Corollary 4.7 tells us that every function $u$ in $\mathcal{D}(L) \subset \mathcal{D}(\bar{A})$ can be written in the form (4.7). Then we define

$$
L u=\overline{L G_{\alpha}^{0}}((\alpha I-\bar{A}) u)+\overline{L H_{\alpha}}\left(\left.u\right|_{\Sigma_{2}}\right) .
$$

The next lemma justifies this definition (see [19, Lemma 4.15]):

LEMma 4.14. The right-hand side of formula (4.11) depends only on u, not on the choice of expression (4.7).

5. Degenerate elliptic boundary value problems. In this section we prove an existence theorem for degenerate elliptic boundary value problems in the framework of Hölder spaces, which will play an important role in the proof of Theorem 1.1 in Section 6.

5.1. Existence theorem for degenerate elliptic boundary value problems. Now we can state our existence theorem for degenerate elliptic boundary value problems in the framework of Hölder spaces:

THEOREM 5.1. Assume that the operator A satisfies hypotheses $(\mathrm{H})$ and (T). Then, for each $\alpha \geq \alpha(10)$, there exists a constant $\lambda=\lambda(\alpha)>0$ such that the boundary value problem

$$
\begin{cases}(\alpha-A) u=f & \text { in } D \\ (\lambda-L) u=\varphi & \text { on } \Sigma_{2},\end{cases}
$$

has a solution $u \in C^{2+\theta}(\bar{D})$ for any $f \in C^{6+2 \theta}(\bar{D})$ and $\varphi \in C^{8+2 \theta}\left(\Sigma_{2}\right)$ with $0<\theta<1$. Here $\alpha(10)$ is the constant appearing in Theorem 4.1 with $m=10$.

5.2. Proof of Theorem 5.1. We divide the proof into three steps.

(I) First we reduce the study of problem $(* *)$ to that of an operator on the boundary $\Sigma_{2}$.

Assume that the following condition is satisfied:

$$
\begin{aligned}
& \text { If } \lambda>0 \text { is sufficiently large, then the operator } \\
& \qquad \lambda I-L H_{\alpha}: C^{8+2 \theta}\left(\Sigma_{2}\right) \rightarrow C^{8+2 \theta}\left(\Sigma_{2}\right)
\end{aligned}
$$

is surjective. 
If $f \in C^{22+2 \theta}(\bar{D})$ and $\varphi \in C^{8+2 \theta}\left(\Sigma_{2}\right)$, then $G_{\alpha}^{0} f \in C^{10+\theta}(\bar{D})$ by Theorem 4.1 with $m=10$, and

$$
L G_{\alpha}^{0} f=\mu \frac{\partial}{\partial \mathbf{n}}\left(G_{\alpha}^{0} f\right)+\delta f \in C^{9+\theta}\left(\Sigma_{2}\right) \subset C^{8+2 \theta}\left(\Sigma_{2}\right) .
$$

Hence, by condition (5.1) one can find $\psi \in C^{8+2 \theta}\left(\Sigma_{2}\right)$ such that

$$
\left(\lambda-L H_{\alpha}\right) \psi=\varphi+L G_{\alpha}^{0} f \quad \text { on } \Sigma_{2} .
$$

If we let

$$
u=G_{\alpha}^{0} f+H_{\alpha} \psi \in C^{2+\theta}(\bar{D}),
$$

then

$$
\begin{cases}(\alpha-A) u=f & \text { in } D \\ (\lambda-L) u=-L G_{\alpha}^{0} f+\left(\lambda-L H_{\alpha}\right) \psi=\varphi & \text { on } \Sigma_{2} .\end{cases}
$$

This proves that $u=G_{\alpha}^{0} f+H_{\alpha} \psi$ is a solution of problem (**).

Therefore we are reduced to the study of the operator $\lambda I-L H_{\alpha}$ on $\Sigma_{2}$.

(II) Secondly we show that $L H_{\alpha}$ is a classical pseudo-differential operator of second order which is the sum of a second-order degenerate elliptic differential operator and a classical pseudo-differential operator of order $2-\kappa$, $\kappa>0$, on the set $\Sigma_{2}$.

From (4.9), we find that

$$
L H_{\alpha} \varphi=P_{\alpha} \varphi+Q_{\alpha} \varphi+S \varphi+R \varphi,
$$

where

$$
\begin{aligned}
P_{\alpha} \varphi & :=\sum_{i, j=1}^{N-1} \alpha^{i j}\left(x^{\prime}\right) \frac{\partial^{2} \varphi}{\partial x_{i} \partial x_{j}}+\sum_{i=1}^{N-1} \beta^{i}\left(x^{\prime}\right) \frac{\partial \varphi}{\partial x_{i}}+\left(\gamma\left(x^{\prime}\right)-\alpha \delta\left(x^{\prime}\right)\right) \varphi \\
Q_{\alpha} \varphi & :=\left(-\frac{\mu\left(x^{\prime}\right)}{b\left(x^{\prime}\right)}\right)\left[\sum_{i, j=1}^{N-1} a^{i j}\left(x^{\prime}\right) \frac{\partial^{2} \varphi}{\partial x_{i} \partial x_{j}}+\sum_{i=1}^{N-1} b^{i}\left(x^{\prime}\right) \frac{\partial \varphi}{\partial x_{i}}+\left(c\left(x^{\prime}\right)-\alpha\right) \varphi\right] \\
S \varphi & :=-\left(\int_{\Sigma_{2}} r\left(x^{\prime}, y^{\prime}\right) d y^{\prime}\right) \varphi-\sum_{j=1}^{N-1}\left(\int_{\Sigma_{2}} r\left(x^{\prime}, y^{\prime}\right)\left(y_{j}-x_{j}\right) d y^{\prime}\right) \frac{\partial \varphi}{\partial x_{j}} \\
R \varphi & :=\int_{\Sigma_{2}} r\left(x^{\prime}, y^{\prime}\right) \varphi\left(y^{\prime}\right) d y^{\prime} .
\end{aligned}
$$

Moreover the operators $P_{\alpha}, Q_{\alpha}, S, R$ satisfy the following conditions (i) through (iv):

(i) $P_{\alpha}$ is a second-order, degenerate elliptic differential operator on $\Sigma_{2}$ with non-positive principal symbol and

$$
P_{\alpha} 1\left(x^{\prime}\right)=\gamma\left(x^{\prime}\right)-\alpha \delta\left(x^{\prime}\right) \leq 0 \quad \text { on } \Sigma_{2} .
$$


(ii) $Q_{\alpha}$ is a second-order, degenerate elliptic differential operator on $\Sigma_{2}$ with non-positive principal symbol and

$$
Q_{\alpha} 1\left(x^{\prime}\right)=-\frac{\mu\left(x^{\prime}\right)}{b\left(x^{\prime}\right)}\left(c\left(x^{\prime}\right)-\alpha\right) \leq 0 \quad \text { on } \Sigma_{2} .
$$

(iii) $R$ is in $L_{1,0}^{2-k}\left(\Sigma_{2}\right)$ and $r\left(x^{\prime}, y^{\prime}\right) \geq 0$ off the diagonal $\Delta_{\Sigma_{2}}$ and so

$$
R 1\left(x^{\prime}\right)=\int_{\Sigma_{2}} r\left(x^{\prime}, y^{\prime}\right) d y^{\prime} \geq 0 \quad \text { on } \Sigma_{2} .
$$

(iv) $S$ is a first-order differential operator on $\Sigma_{2}$ and

$$
S 1\left(x^{\prime}\right)=-\int_{\Sigma_{2}} r\left(x^{\prime}, y^{\prime}\right) d y^{\prime} \leq 0 \quad \text { on } \Sigma_{2} .
$$

(III) Since

$$
\begin{aligned}
L H_{\alpha} 1\left(x^{\prime}\right) & =P_{\alpha} 1\left(x^{\prime}\right)+Q_{\alpha} 1\left(x^{\prime}\right)+S 1\left(x^{\prime}\right)+R 1\left(x^{\prime}\right) \\
& =\gamma\left(x^{\prime}\right)-\alpha \delta\left(x^{\prime}\right)-\frac{\mu\left(x^{\prime}\right)}{b\left(x^{\prime}\right)}\left(c\left(x^{\prime}\right)-\alpha\right) \leq 0 \quad \text { on } \Sigma_{2},
\end{aligned}
$$

we can apply Theorem 3.1 with $P:=P_{\alpha}+Q_{\alpha}+S$ and $S:=R$ to deduce that the operator

$$
\lambda I-L H_{\alpha}: C^{8+2 \theta}\left(\Sigma_{2}\right) \rightarrow C^{8+2 \theta}\left(\Sigma_{2}\right)
$$

is surjective for $\lambda>0$ sufficiently large, that is, assertion (5.1) is proved.

The proof of Theorem 5.1 is now complete.

6. Proof of Theorem 1.1. First we reduce the problem of construction of Feller semigroups to the problem of unique solvability for the boundary value problem $(* *)_{0}$, and then prove existence theorems for Feller semigroups. The proof is divided into seven steps.

(I) First we prove that

(6.1) the operator $\overline{L H_{\alpha}}$ generates a Feller semigroup on $\Sigma_{2}$ for any $\alpha>0$.

By Theorem 5.1, for each $\alpha \geq \alpha(10)$ one can find a constant $\lambda=\lambda(\alpha)>0$ such that the boundary value problem $(* *)_{0}$ has a solution $u \in C^{2+\theta}(\bar{D})$ for any $\varphi \in C^{8+2 \theta}\left(\Sigma_{2}\right)$. Therefore (6.1) follows from Theorem 4.13.

(II) Next we prove that

(6.2) the equation $\overline{L H_{\alpha}} \psi=\varphi$ has a unique solution $\psi \in \mathcal{D}\left(\overline{L H_{\alpha}}\right)$ for any $\varphi \in C\left(\Sigma_{2}\right)$; hence the inverse ${\overline{L H_{\alpha}}}^{-1}$ can be defined on the whole $C\left(\Sigma_{2}\right)$. Moreover it is non-negative and bounded on $C\left(\Sigma_{2}\right)$.

By (4.9) with $\varphi:=1$, it follows that

$$
\frac{\partial}{\partial \mathbf{n}}\left(H_{\alpha} 1\right)\left(x^{\prime}\right)=-\frac{c\left(x^{\prime}\right)-\alpha}{b\left(x^{\prime}\right)}<0 \quad \text { on } \Sigma_{2} .
$$


Thus transversality condition $(\mathrm{T})$ implies that

$$
L H_{\alpha} 1\left(x^{\prime}\right)=\mu\left(x^{\prime}\right) \frac{\partial}{\partial \mathbf{n}}\left(H_{\alpha} 1\right)\left(x^{\prime}\right)+\gamma\left(x^{\prime}\right)-\alpha \delta\left(x^{\prime}\right)<0 \quad \text { on } \Sigma_{2},
$$

and so

$$
\ell_{\alpha}=-\max _{x^{\prime} \in \Sigma_{2}} L H_{\alpha} 1\left(x^{\prime}\right)>0 .
$$

Furthermore, using Corollary 2.4 with $K:=\Sigma_{2}, \mathfrak{A}:=\overline{L H_{\alpha}}$ and $c:=\ell_{\alpha}$ we find that $\overline{L H_{\alpha}}+\ell_{\alpha} I$ is the infinitesimal generator of a Feller semigroup on $\Sigma_{2}$. Therefore, since $\ell_{\alpha}>0$, an application of Theorem 2.3(i) with $\mathfrak{A}:=$ $\overline{L H_{\alpha}}+\ell_{\alpha} I$ shows that the equation

$$
-\overline{L H_{\alpha}} \psi=\left(\ell_{\alpha} I-\left(\overline{L H_{\alpha}}+\ell_{\alpha} I\right)\right) \psi=\varphi
$$

has a unique solution $\psi \in \mathcal{D}\left(\overline{L H_{\alpha}}\right)$ for any $\varphi \in C\left(\Sigma_{2}\right)$, and the operator $-{\overline{L H_{\alpha}}}^{-1}=\left(\ell_{\alpha} I-\left(\overline{L H_{\alpha}}+\ell_{\alpha} I\right)\right)^{-1}$ is non-negative and bounded on $C\left(\Sigma_{2}\right)$ with norm

$$
\left\|-{\overline{L H_{\alpha}}}^{-1}\right\|=\left\|\left(\ell_{\alpha} I-\left(\overline{L H_{\alpha}}+\ell_{\alpha} I\right)\right)^{-1}\right\| \leq 1 / \ell_{\alpha} .
$$

(III) By (6.2), we can define, for all $\alpha>0$,

$$
G_{\alpha} f=G_{\alpha}^{0} f-H_{\alpha}\left({\overline{L H_{\alpha}}}^{-1}\left(\overline{L G_{\alpha}^{0}} f\right)\right), \quad f \in C(\bar{D}) .
$$

Now we prove that

$$
G_{\alpha}=(\alpha I-\mathfrak{A})^{-1} .
$$

In view of Lemmas 4.6 and 4.12,

$$
\left\{\begin{array}{l}
G_{\alpha} f=G_{\alpha}^{0} f-H_{\alpha}\left({\overline{L H_{\alpha}}}^{-1}\left(\overline{L G_{\alpha}^{0}} f\right)\right) \in \mathcal{D}(\bar{A}), \\
\left.G_{\alpha} f\right|_{\Sigma_{2}}=-\overline{L H}_{\alpha}-1\left(\overline{L G_{\alpha}^{0}} f\right) \in \mathcal{D}\left(\overline{L H_{\alpha}}\right)=\mathcal{D}, \\
L G_{\alpha} f=\overline{L G_{\alpha}^{0}} f-\overline{L H_{\alpha}}\left(\overline{L H_{\alpha}}-1\left(\overline{L G_{\alpha}^{0}} f\right)\right)=0,
\end{array}\right.
$$

and

$$
(\alpha I-\bar{A}) G_{\alpha} f=f .
$$

That is, for any $f \in C(\bar{D})$,

$$
\left\{\begin{array}{l}
G_{\alpha} f \in \mathcal{D}(\mathfrak{A}) \\
(\alpha I-\mathfrak{A}) G_{\alpha} f=f
\end{array}\right.
$$

so that

$$
(\alpha I-\mathfrak{A}) G_{\alpha}=I \quad \text { on } C(\bar{D}) .
$$

Therefore, in order to prove (6.5) it suffices to show the injectivity of $\alpha I-\mathfrak{A}$.

Assume that $u \in \mathcal{D}(\mathfrak{A})$ and $(\alpha I-\mathfrak{A}) u=0$. Then, by Corollary 4.7,

$$
u=H_{\alpha}\left(\left.u\right|_{\Sigma_{2}}\right),\left.\quad u\right|_{\Sigma_{2}} \in \mathcal{D}=\mathcal{D}\left(\overline{L H_{\alpha}}\right) .
$$

Thus $\overline{L H_{\alpha}}\left(\left.u\right|_{\Sigma_{2}}\right)=L u=0$. In view of $(6.2)$, this implies that $\left.u\right|_{\Sigma_{2}}=0$, so that $u=H_{\alpha}\left(\left.u\right|_{\Sigma_{2}}\right)=0$ in $D$. 
(IV) The non-negativity of $G_{\alpha}$ follows immediately from formula (6.4), since the operators $G_{\alpha}^{0}, H_{\alpha},-{\overline{L H_{\alpha}}}^{-1}$ and $\overline{L G_{\alpha}^{0}}$ are all non-negative.

(V) We prove that $G_{\alpha}$ is bounded on $C(\bar{D})$ with norm

$$
\left\|G_{\alpha}\right\| \leq 1 / \alpha .
$$

It suffices to show that

$$
G_{\alpha} 1 \leq 1 / \alpha \quad \text { on } \bar{D}
$$

since $G_{\alpha}$ is non-negative on $C(\bar{D})$.

From the uniqueness theorem for problem (D) (Theorem 4.1) it follows that

$$
\alpha G_{\alpha}^{0} 1(x)+H_{\alpha} 1(x)=1+G_{\alpha}^{0} c(x) \quad \text { on } \bar{D} \text {. }
$$

Indeed, both sides have the same boundary value 1 on $\Sigma_{2}$ and satisfy the same equation: $(\alpha I-A) u=\alpha$ in $D$.

Applying the operator $L$ to both sides of (6.7), we obtain

$$
\begin{aligned}
-L H_{\alpha} 1\left(x^{\prime}\right) & =-L 1\left(x^{\prime}\right)-L G_{\alpha}^{0} c\left(x^{\prime}\right)+\alpha L G_{\alpha}^{0} 1\left(x^{\prime}\right) \\
& =-\mu\left(x^{\prime}\right) \frac{\partial}{\partial \mathbf{n}}\left(G_{\alpha}^{0} c\right)\left(x^{\prime}\right)+\alpha L G_{\alpha}^{0} 1\left(x^{\prime}\right)-\gamma\left(x^{\prime}\right) \\
& \geq \alpha L G_{\alpha}^{0} 1\left(x^{\prime}\right) \quad \text { on } \Sigma_{2},
\end{aligned}
$$

since $G_{\alpha}^{0} c\left(x^{\prime}\right)=0$ and $G_{\alpha}^{0} c(x) \leq 0$ on $\bar{D}$. Hence, by the non-negativity of $-{\overline{L H_{\alpha}}}^{-1}$,

$$
-{\overline{L H_{\alpha}}}^{-1}\left(L G_{\alpha}^{0} 1\right) \leq 1 / \alpha \quad \text { on } \Sigma_{2} .
$$

Using (6.4) with $f:=1,(6.8)$ and (6.7), we find that

$$
\begin{aligned}
G_{\alpha} 1 & =G_{\alpha}^{0} 1+H_{\alpha}\left(-{\overline{L H_{\alpha}}}^{-1}\left(L G_{\alpha}^{0} 1\right)\right) \leq G_{\alpha}^{0} 1+\frac{1}{\alpha} H_{\alpha} 1 \\
& =\frac{1}{\alpha}+\frac{1}{\alpha} G_{\alpha}^{0} c \leq \frac{1}{\alpha} \quad \text { on } \bar{D},
\end{aligned}
$$

since the operators $H_{\alpha}$ and $G_{\alpha}^{0}$ are non-negative and $c(x) \leq 0$ on $D$.

(VI) Finally we prove that

$$
\text { the domain } \mathcal{D}(\mathfrak{A}) \text { is dense in } C(\bar{D}) \text {. }
$$

(VI-1) Before the proof, we need some lemmas on the behavior of the operators $G_{\alpha}^{0}, H_{\alpha}$ and $-{\overline{L H_{\alpha}}}^{-1}$ as $\alpha \rightarrow+\infty$ :

Lemma 6.1. For all $f \in C(\bar{D})$, we have

$$
\lim _{\alpha \rightarrow+\infty}\left[\alpha G_{\alpha}^{0} f+H_{\alpha}\left(\left.f\right|_{\Sigma_{2}}\right)\right]=f \quad \text { in } C(\bar{D}) .
$$

Proof. Choose a constant $\beta>0$ and let

$$
g=f-H_{\beta}\left(\left.f\right|_{\Sigma_{2}}\right) .
$$


Then, using (4.6) with $\varphi:=\left.f\right|_{\Sigma_{2}}$, we obtain

$$
\alpha G_{\alpha}^{0} g-g=\left[\alpha G_{\alpha}^{0} f+H_{\alpha}\left(\left.f\right|_{\Sigma_{2}}\right)-f\right]-\beta G_{\alpha}^{0} H_{\beta}\left(\left.f\right|_{\Sigma_{2}}\right) .
$$

However we have, by estimate (4.3),

and by $\left(4.5^{\prime}\right)$,

$$
\lim _{\alpha \rightarrow+\infty} G_{\alpha}^{0} H_{\beta}\left(\left.f\right|_{\Sigma_{2}}\right)=0 \quad \text { in } C(\bar{D})
$$

$$
\lim _{\alpha \rightarrow+\infty} \alpha G_{\alpha}^{0} g=g \quad \text { in } C(\bar{D}),
$$

since $\left.g\right|_{\Sigma_{2}}=0$. Therefore (6.10) follows by letting $\alpha \rightarrow+\infty$ in (6.11).

Lemma 6.2. $\lim _{\alpha \rightarrow+\infty}\left\|-{\overline{L H_{\alpha}}}^{-1}\right\|=0$.

Proof. Since $\mu\left(x^{\prime}\right)+\delta\left(x^{\prime}\right)>0$ on $\Sigma_{2}$, we find from (4.9) with $\varphi:=1$ that the function

$$
\begin{aligned}
L H_{\alpha} 1\left(x^{\prime}\right) & =\mu\left(x^{\prime}\right) \frac{\partial}{\partial \mathbf{n}}\left(H_{\alpha} 1\right)\left(x^{\prime}\right)+\gamma\left(x^{\prime}\right)-\alpha \delta\left(x^{\prime}\right) \\
& =\mu\left(x^{\prime}\right)\left[-\frac{1}{b\left(x^{\prime}\right)}\left(c\left(x^{\prime}\right)-\alpha\right)\right]+\gamma\left(x^{\prime}\right)-\alpha \delta\left(x^{\prime}\right) \\
& =-\alpha\left(\frac{\mu\left(x^{\prime}\right)}{-b\left(x^{\prime}\right)}+\delta\left(x^{\prime}\right)\right)+\gamma\left(x^{\prime}\right)-\frac{\mu\left(x^{\prime}\right) c\left(x^{\prime}\right)}{b\left(x^{\prime}\right)}
\end{aligned}
$$

diverges to $-\infty$ monotonically as $\alpha \rightarrow+\infty$. By Dini's theorem, this convergence is uniform in $x^{\prime} \in \Sigma_{2}$. Hence $1 / L H_{\alpha} 1\left(x^{\prime}\right) \rightarrow 0$ uniformly in $x^{\prime} \in \Sigma_{2}$ as $\alpha \rightarrow+\infty$. This implies that

$$
\left\|-{\overline{L H_{\alpha}}}^{-1}\right\|=\left\|-{\overline{L H_{\alpha}}}^{-1} 1\right\|_{\infty} \leq\left\|\frac{1}{L H_{\alpha} 1}\right\|_{\infty} \rightarrow 0 \quad \text { as } \alpha \rightarrow+\infty .
$$

(VI-2) Proof of assertion (6.9). In view of (6.5) and (6.6), it suffices to prove that

$$
\lim _{\alpha \rightarrow+\infty}\left\|\alpha G_{\alpha} f-f\right\|_{\infty}=0, \quad f \in C^{\infty}(\bar{D}),
$$

since $C^{\infty}(\bar{D})$ is dense in $C(\bar{D})$.

First we remark that

$$
\begin{aligned}
\left\|\alpha G_{\alpha} f-f\right\|_{\infty}= & \left\|\alpha G_{\alpha}^{0} f-\alpha H_{\alpha}\left({\overline{L H_{\alpha}}}^{-1}\left(L G_{\alpha}^{0} f\right)\right)-f\right\|_{\infty} \\
\leq & \left\|\alpha G_{\alpha}^{0} f+H_{\alpha}\left(\left.f\right|_{\Sigma_{2}}\right)-f\right\|_{\infty} \\
& +\left\|-\alpha H_{\alpha}\left(\overline{L H}_{\alpha}^{-1}\left(L G_{\alpha}^{0} f\right)\right)-H_{\alpha}\left(\left.f\right|_{\Sigma_{2}}\right)\right\|_{\infty} \\
\leq & \left\|\alpha G_{\alpha}^{0} f+H_{\alpha}\left(\left.f\right|_{\Sigma_{2}}\right)-f\right\|_{\infty} \\
& +\left\|-\alpha{\overline{L H_{\alpha}}}^{-1}\left(L G_{\alpha}^{0} f\right)-\left.f\right|_{\Sigma_{2}}\right\|_{\infty} .
\end{aligned}
$$

Thus, in view of (6.10) it suffices to show that

$$
\lim _{\alpha \rightarrow+\infty}\left[\alpha{\overline{L H_{\alpha}}}^{-1} L G_{\alpha}^{0} f+\left.f\right|_{\Sigma_{2}}\right]=0 \quad \text { in } C\left(\Sigma_{2}\right) .
$$


Take a constant $\beta$ such that $0<\beta<\alpha$, and write

$$
f=G_{\beta}^{0} g+H_{\beta} \varphi
$$

where (see formula (4.7))

$$
\left\{\begin{array}{l}
g=(\beta-A) f \in C^{\infty}(\bar{D}) \\
\varphi=\left.f\right|_{\Sigma_{2}} \in C^{\infty}\left(\Sigma_{2}\right)
\end{array}\right.
$$

Then, using (4.4) (with $f:=g$ ) and (4.6) we obtain

$$
G_{\alpha}^{0} f=G_{\alpha}^{0} G_{\beta}^{0} g+G_{\alpha}^{0} H_{\beta} \varphi=\frac{1}{\alpha-\beta}\left(G_{\beta}^{0} g-G_{\alpha}^{0} g+H_{\beta} \varphi-H_{\alpha} \varphi\right) .
$$

Hence

$$
\begin{aligned}
\|- & \alpha{\overline{L H_{\alpha}}}^{-1}\left(L G_{\alpha}^{0} f\right)-\left.f\right|_{\Sigma_{2}} \|_{\infty} \\
& =\left\|\frac{\alpha}{\alpha-\beta}\left(-{\overline{L H_{\alpha}}}^{-1}\right)\left(L G_{\beta}^{0} g-L G_{\alpha}^{0} g+L H_{\beta} \varphi\right)+\frac{\alpha}{\alpha-\beta} \varphi-\varphi\right\|_{\infty} \\
& \leq \frac{\alpha}{\alpha-\beta}\left\|-{\overline{L H_{\alpha}}}^{-1}\right\| \cdot\left\|L G_{\beta}^{0} g+L H_{\beta} \varphi\right\|_{\infty} \\
& +\frac{\alpha}{\alpha-\beta}\left\|-{\overline{L H_{\alpha}}}^{-1}\right\| \cdot\left\|L G_{\alpha}^{0}\right\| \cdot\|g\|_{\infty}+\frac{\beta}{\alpha-\beta}\|\varphi\|_{\infty} .
\end{aligned}
$$

By Lemma 6.2, the first term on the right-hand side converges to zero as $\alpha \rightarrow+\infty$. For the second term, using (4.4) with $f:=1$ and the nonnegativity of $G_{\beta}^{0}$ and $L G_{\alpha}^{0}$ we find that

$$
\left\|L G_{\alpha}^{0}\right\|=\left\|L G_{\alpha}^{0} 1\right\|_{\infty}=\left\|L G_{\beta}^{0} 1-(\alpha-\beta) L G_{\alpha}^{0} G_{\beta}^{0} 1\right\|_{\infty} \leq\left\|L G_{\beta}^{0} 1\right\|_{\infty} .
$$

Hence the second term also converges to zero as $\alpha \rightarrow+\infty$, and it is clear that so does the third term. This completes the proof of (6.13) and hence of (6.12).

(VII) Summing up, we have proved that the operator $\mathfrak{A}$ of Theorem 1.1 satisfies conditions (a) through (d) of Theorem 2.3. Hence $\mathfrak{A}$ is the infinitesimal generator of some Feller semigroup on $\bar{D}$.

The proof of Theorem 1.1 is now complete.

7. Proof of Theorem 1.2. First we show that if condition (B) of Section 1 is satisfied, then the operator $L H_{\alpha}$ is bijective in Hölder spaces. This is proved by applying Theorem 2.3 just as in the proof of Theorem 1.1. Therefore the unique solution $u$ of the boundary value problem $(*)$ with $\varphi=0$ can be expressed in the form

$$
u=G_{\alpha} f=G_{\alpha}^{\nu} f-H_{\alpha}\left({\overline{L H_{\alpha}}}^{-1}\left(L G_{\alpha}^{\nu} f\right)\right) .
$$

This formula allows us to verify all the conditions of the generation theorems for Feller semigroups discussed in Subsection 2.2. 
First we simplify the boundary condition

$$
L u\left(x^{\prime}\right)=0 \quad \text { on } \Sigma_{2} .
$$

By conditions (A) and (B), one may assume that

$$
L u\left(x^{\prime}\right)=m\left(x^{\prime}\right) L_{\nu} u\left(x^{\prime}\right)+\left(m\left(x^{\prime}\right)-1\right) u\left(x^{\prime}\right),
$$

with

$$
0 \leq m\left(x^{\prime}\right) \leq 1 \quad \text { on } \Sigma_{2} .
$$

Indeed, it suffices to note that the boundary condition

$$
L u\left(x^{\prime}\right)=m\left(x^{\prime}\right) L_{\nu} u\left(x^{\prime}\right)+\gamma\left(x^{\prime}\right) u\left(x^{\prime}\right)=0 \quad \text { on } \Sigma_{2}
$$

is equivalent to the condition

$$
\left(\frac{m\left(x^{\prime}\right)}{m\left(x^{\prime}\right)-\gamma\left(x^{\prime}\right)}\right) L_{\nu} u\left(x^{\prime}\right)+\left(\frac{\gamma\left(x^{\prime}\right)}{m\left(x^{\prime}\right)-\gamma\left(x^{\prime}\right)}\right) u\left(x^{\prime}\right)=0 \quad \text { on } \Sigma_{2} .
$$

Furthermore we remark that

$$
\overline{L G_{\alpha}^{0}} f\left(x^{\prime}\right)=m\left(x^{\prime}\right) \overline{L_{\nu} G_{\alpha}^{0}} f\left(x^{\prime}\right)
$$

and

$$
\overline{L H_{\alpha}} \varphi\left(x^{\prime}\right)=m\left(x^{\prime}\right) \overline{L_{\nu} H_{\alpha}} \varphi\left(x^{\prime}\right)+\left(m\left(x^{\prime}\right)-1\right) \varphi\left(x^{\prime}\right) .
$$

Hence, in view of definition (4.11) it follows that

$$
L u\left(x^{\prime}\right)=m\left(x^{\prime}\right) L_{\nu} u\left(x^{\prime}\right)+\left(m\left(x^{\prime}\right)-1\right) u\left(x^{\prime}\right), \quad u \in \mathcal{D}(L) .
$$

Therefore the next theorem proves Theorem 1.2:

TheOrem 7.1. Define a linear operator

$$
\mathfrak{A}_{0}: C_{0}(\bar{D} \backslash M) \rightarrow C_{0}(\bar{D} \backslash M)
$$

by setting

$$
\begin{aligned}
\mathcal{D}\left(\mathfrak{A}_{0}\right)=\left\{u \in C_{0}(\bar{D} \backslash M): \bar{A} u \in C_{0}(\bar{D} \backslash M),\right. \\
\left.L u=m L_{\nu} u+(m-1)\left(\left.u\right|_{\Sigma_{2}}\right)=0\right\},
\end{aligned}
$$

and

$$
\mathfrak{A}_{0} u=\bar{A} u, \quad u \in \mathcal{D}\left(\mathfrak{A}_{0}\right) .
$$

Assume that

$$
0 \leq m\left(x^{\prime}\right) \leq 1 \quad \text { on } \Sigma_{2} .
$$

Then the operator $\mathfrak{A}_{0}$ generates some Feller semigroup $\left\{U_{t}\right\}_{t \geq 0}$ on $\bar{D} \backslash M$ and the Green operator $G_{\alpha}=\left(\alpha I-\mathfrak{A}_{0}\right)^{-1}, \alpha>0$, is given by the formula

$$
G_{\alpha} f=G_{\alpha}^{\nu} f-H_{\alpha}\left({\overline{L H_{\alpha}}}^{-1}\left(L G_{\alpha}^{\nu} f\right)\right), \quad f \in C_{0}(\bar{D} \backslash M) .
$$

Here $G_{\alpha}^{\nu}$ is the Green operator for the boundary condition $L_{\nu}$ given by (6.4):

$$
G_{\alpha}^{\nu} f=G_{\alpha}^{0} f-H_{\alpha}\left({\overline{L_{\nu} H_{\alpha}}}^{-1}\left(\overline{L_{\nu} G_{\alpha}^{0}} f\right)\right), \quad f \in C(\bar{D}) .
$$


Proof. We apply Theorem 2.3(ii) to the operator $\mathfrak{A}_{0}$ defined by $(7.2)$ and (7.3), just as in the proof of Theorem 1.1. The proof consists of five steps.

(I) First we prove that, for all $\alpha>0$, the operator $\overline{L H_{\alpha}}$ generates a Feller semigroup on $\Sigma_{2}$.

To this end, we remark (cf. Claim 4.11) that

$$
\begin{aligned}
L H_{\alpha} \varphi\left(x^{\prime}\right)= & m\left(x^{\prime}\right) L_{\nu} H_{\alpha} \varphi\left(x^{\prime}\right)+\left(m\left(x^{\prime}\right)-1\right) \varphi\left(x^{\prime}\right) \\
= & m\left(x^{\prime}\right)\left(\sum_{i, j=1}^{N-1} \bar{\alpha}^{i j} \frac{\partial^{2} \varphi}{\partial x_{i} \partial x_{j}}\left(x^{\prime}\right)+\sum_{i=1}^{N-1} \bar{\beta}^{i}\left(x^{\prime}\right) \frac{\partial \varphi}{\partial x_{i}}\left(x^{\prime}\right)\right) \\
& +\left(\left(m\left(x^{\prime}\right)-1\right)-\alpha m\left(x^{\prime}\right) \bar{\delta}\left(x^{\prime}\right)\right) \varphi\left(x^{\prime}\right)+m\left(x^{\prime}\right) \bar{\mu}\left(x^{\prime}\right) \frac{\partial}{\partial \mathbf{n}}\left(H_{\alpha} \varphi\right)\left(x^{\prime}\right) \\
& +m\left(x^{\prime}\right) \int_{\Sigma_{2}} \bar{r}\left(x^{\prime}, y^{\prime}\right)\left[\varphi\left(y^{\prime}\right)-\varphi\left(x^{\prime}\right)-\sum_{j=1}^{N-1}\left(y_{j}-x_{j}\right) \frac{\partial \varphi}{\partial x_{j}}\left(x^{\prime}\right)\right] d y^{\prime} .
\end{aligned}
$$

Furthermore, as in inequality (6.3), we have

$$
\frac{\partial}{\partial \mathbf{n}}\left(H_{\alpha} 1\right)\left(x^{\prime}\right)<0 \quad \text { on } \Sigma_{2} .
$$

By conditions $\left(\mathrm{A}^{\prime}\right)$ and $(\overline{\mathrm{T}})$, this implies that

$$
\begin{aligned}
L H_{\alpha} 1\left(x^{\prime}\right) & =m\left(x^{\prime}\right) L_{\nu} H_{\alpha} 1\left(x^{\prime}\right)+\left(m\left(x^{\prime}\right)-1\right) \\
& =m\left(x^{\prime}\right)\left(\bar{\mu}\left(x^{\prime}\right) \frac{\partial}{\partial \mathbf{n}}\left(H_{\alpha} 1\right)\left(x^{\prime}\right)-\alpha \bar{\delta}\left(x^{\prime}\right)\right)+\left(m\left(x^{\prime}\right)-1\right) \\
& <0 \quad \text { on } \Sigma_{2} .
\end{aligned}
$$

Then, applying Theorem 3.1 to the operator $L H_{\alpha}$ (see the proof of (5.1)), we find that if $\lambda>0$ is sufficiently large, then $\mathcal{R}\left(\lambda I-L H_{\alpha}\right) \supset C^{8+2 \theta}\left(\Sigma_{2}\right)$. In particular, $\mathcal{R}\left(\lambda I-L H_{\alpha}\right)$ is dense in $C\left(\Sigma_{2}\right)$.

Therefore, Theorem 2.5(ii) applied to $L$ shows that $\overline{L H_{\alpha}}$ is the infinitesimal generator of a Feller semigroup on $\Sigma_{2}$, for all $\alpha>0$.

(II) Now we prove that if condition $\left(\mathrm{A}^{\prime}\right)$ is satisfied, then

(7.6) the equation $\overline{L H_{\alpha}} \psi=\varphi$ has a unique solution $\psi \in \mathcal{D}\left(\overline{L H_{\alpha}}\right)$ for any $\varphi \in C\left(\Sigma_{2}\right)$; hence the inverse $\overline{L H}_{\alpha}^{-1}$ can be defined on the whole space $C\left(\Sigma_{2}\right)$. Moreover it is non-negative and bounded on $C\left(\Sigma_{2}\right)$.

Since by $(7.5)$ and conditions $\left(\mathrm{A}^{\prime}\right)$ and $(\overline{\mathrm{T}})$ we have

$$
\begin{aligned}
L H_{\alpha} 1\left(x^{\prime}\right) & =m\left(x^{\prime}\right) L_{\nu} H_{\alpha} 1\left(x^{\prime}\right)+\left(m\left(x^{\prime}\right)-1\right) \\
& =m\left(x^{\prime}\right)\left(\bar{\mu}\left(x^{\prime}\right) \frac{\partial}{\partial \mathbf{n}}\left(H_{\alpha} 1\right)\left(x^{\prime}\right)-\alpha \bar{\delta}\left(x^{\prime}\right)\right)+\left(m\left(x^{\prime}\right)-1\right) \\
& <0 \quad \text { on } \Sigma_{2},
\end{aligned}
$$


it follows that

$$
k_{\alpha}=-\max _{x^{\prime} \in \Sigma_{2}} L H_{\alpha} 1\left(x^{\prime}\right)>0 .
$$

Here we remark that the constants $k_{\alpha}$ are increasing in $\alpha>0$ :

$$
\alpha \geq \beta>0 \Rightarrow k_{\alpha} \geq k_{\beta}
$$

Moreover, using Corollary 2.4 with $K:=\Sigma_{2}, A:=\overline{L H_{\alpha}}$ and $c:=k_{\alpha}$, we see that the operator $\overline{L H_{\alpha}}+k_{\alpha} I$ is the infinitesimal generator of a Feller semigroup on $\Sigma_{2}$. Therefore, since $k_{\alpha}>0$, an application of Theorem 2.3(i) with $A:=\overline{L H_{\alpha}}+k_{\alpha} I$ shows that the equation

$$
-\overline{L H_{\alpha}} \psi=\left(k_{\alpha} I-\left(\overline{L H_{\alpha}}+k_{\alpha} I\right)\right) \psi=\varphi
$$

has a unique solution $\psi \in \mathcal{D}\left(\overline{L H_{\alpha}}\right)$ for any $\varphi \in C\left(\Sigma_{2}\right)$ and further that the operator

$$
-{\overline{L H_{\alpha}}}^{-1}=\left(k_{\alpha} I-\left(\overline{L H_{\alpha}}+k_{\alpha} I\right)\right)^{-1}
$$

is non-negative and bounded on $C\left(\Sigma_{2}\right)$ with norm

$$
\left\|-{\overline{L H_{\alpha}}}^{-1}\right\|=\left\|\left(k_{\alpha} I-\left(\overline{L H_{\alpha}}+k_{\alpha} I\right)\right)^{-1}\right\| \leq 1 / k_{\alpha} .
$$

(III) By (7.6), we can define the operator $G_{\alpha}$ by (7.4). We prove that

$$
G_{\alpha}=\left(\alpha I-\mathfrak{A}_{0}\right)^{-1}, \quad \alpha>0 .
$$

By Lemma 4.6 and (6.4), it follows that, for all $f \in C_{0}(\bar{D} \backslash M)$,

$$
G_{\alpha} f \in \mathcal{D}(\bar{A}) \text { and } \quad \bar{A} G_{\alpha} f=\alpha G_{\alpha} f-f .
$$

Further we have

$$
L G_{\alpha} f=L G_{\alpha}^{\nu} f-\overline{L H_{\alpha}}\left({\overline{L H_{\alpha}}}^{-1}\left(L G_{\alpha}^{\nu} f\right)\right)=0 \quad \text { on } \Sigma_{2} .
$$

However we recall that

$$
L u=m L_{\nu} u+(m-1)\left(\left.u\right|_{\Sigma_{2}}\right), \quad u \in \mathcal{D}(L) .
$$

This implies that

$$
G_{\alpha} f\left(x^{\prime}\right)=0 \quad \text { on } M=\left\{x^{\prime} \in \Sigma_{2}: m\left(x^{\prime}\right)=0\right\}
$$

and so

$$
\bar{A} G_{\alpha} f\left(x^{\prime}\right)=\alpha G_{\alpha} f\left(x^{\prime}\right)-f\left(x^{\prime}\right)=0 \quad \text { on } M .
$$

Summing up, we have proved that $G_{\alpha} f \in \mathcal{D}\left(\mathfrak{A}_{0}\right)$, and $\left(\alpha I-\mathfrak{A}_{0}\right) G_{\alpha} f=f$. This implies that

$$
\left(\alpha I-\mathfrak{A}_{0}\right) G_{\alpha}=I \quad \text { on } C_{0}(\bar{D} \backslash M) .
$$

Therefore, in order to prove (7.8) it suffices to show the injectivity of $\alpha I-\mathfrak{A}_{0}$ for $\alpha>0$.

Assume that $u \in \mathcal{D}\left(\mathfrak{A}_{0}\right)$ and $\left(\alpha I-\mathfrak{A}_{0}\right) u=0$. Then, by Corollary 4.7,

$$
u=H_{\alpha}\left(\left.u\right|_{\Sigma_{2}}\right),\left.\quad u\right|_{\Sigma_{2}} \in \mathcal{D}\left(\overline{L H_{\alpha}}\right) .
$$


Thus we have $\overline{L H_{\alpha}}\left(\left.u\right|_{\Sigma_{2}}\right)=L u=0$ on $\Sigma_{2}$. By (7.6), this implies that $\left.u\right|_{\Sigma_{2}}=0$, so that $u=H_{\alpha}\left(\left.u\right|_{\Sigma_{2}}\right)=0$ in $D$.

Now we prove the following three assertions:

(i) The operator $G_{\alpha}$ is non-negative on $C_{0}(\bar{D} \backslash M)$ :

$f \in C_{0}(\bar{D} \backslash M), f(x) \geq 0$ on $\bar{D} \backslash M \Rightarrow G_{\alpha} f(x) \geq 0$ on $\bar{D} \backslash M$.

(ii) The operator $G_{\alpha}$ is bounded on $C_{0}(\bar{D} \backslash M)$ with norm

$$
\left\|G_{\alpha}\right\| \leq 1 / \alpha, \quad \alpha>0 .
$$

(iii) The domain $\mathcal{D}\left(\mathfrak{A}_{0}\right)$ is dense in the space $C_{0}(\bar{D} \backslash M)$.

(i) First we show the non-negativity of $G_{\alpha}$ on $C(\bar{D})$ :

$$
f \in C(\bar{D}), f(x) \geq 0 \text { on } \bar{D} \Rightarrow G_{\alpha} f(x) \geq 0 \text { on } \bar{D} .
$$

Recall that the Dirichlet problem

$$
\begin{cases}(\alpha I-A) u=f & \text { in } D \\ \left.u\right|_{\Sigma_{2}}=\varphi & \text { on } \Sigma_{2},\end{cases}
$$

is uniquely solvable. Hence

$$
G_{\alpha}^{\nu} f=H_{\alpha}\left(\left.G_{\alpha}^{\nu} f\right|_{\Sigma_{2}}\right)+G_{\alpha}^{0} f \quad \text { on } \bar{D},
$$

since both sides have the same boundary values and satisfy the same equation $(\alpha I-A) u=f$ in $D$. Thus, applying $L$ to both sides of (7.10) we obtain

$$
L G_{\alpha}^{\nu} f=\overline{L H_{\alpha}}\left(\left.G_{\alpha}^{\nu} f\right|_{\Sigma_{2}}\right)+\overline{L G_{\alpha}^{0}} f .
$$

Since the operators $-{\overline{L H_{\alpha}}}^{-1}$ and $\overline{L G_{\alpha}^{0}}$ are non-negative, it follows that $\left(-{\overline{L H_{\alpha}}}^{-1}\left(L G_{\alpha}^{\nu} f\right)\right)=-\left.G_{\alpha}^{\nu} f\right|_{\Sigma_{2}}+\left(-{\overline{L H_{\alpha}}}^{-1}\left(\overline{L G_{\alpha}^{0}} f\right)\right) \geq-\left.G_{\alpha}^{\nu} f\right|_{\Sigma_{2}} \quad$ on $\Sigma_{2}$.

Therefore, by the non-negativity of $H_{\alpha}$ and $G_{\alpha}^{0}$ we find that

$$
\begin{aligned}
G_{\alpha} f & =G_{\alpha}^{\nu} f+H_{\alpha}\left(-{\overline{L H_{\alpha}}}^{-1}\left(L G_{\alpha}^{\nu} f\right)\right) \geq G_{\alpha}^{\nu} f-H_{\alpha}\left(\left.G_{\alpha}^{\nu} f\right|_{\Sigma_{2}}\right) \\
& =G_{\alpha}^{0} f \geq 0 \quad \text { on } \bar{D} .
\end{aligned}
$$

(ii) Next we prove the boundedness of $G_{\alpha}$ on $C_{0}(\bar{D} \backslash M)$ with

$$
\left\|G_{\alpha}\right\| \leq 1 / \alpha, \quad \alpha>0 .
$$

To this end, it suffices to show that

$$
f \in C_{0}(\bar{D} \backslash M), f(x) \geq 0 \text { on } \bar{D} \Rightarrow \alpha G_{\alpha} f(x) \leq \max _{\bar{D}} f \text { on } \bar{D},
$$

since $G_{\alpha}$ is non-negative on $C(\bar{D})$.

We remark (cf. $\left.\left(7.1^{\prime}\right)\right)$ that

$$
\begin{aligned}
L G_{\alpha}^{\nu} f\left(x^{\prime}\right) & =m\left(x^{\prime}\right) L_{\nu} G_{\alpha}^{\nu} f\left(x^{\prime}\right)+\left(m\left(x^{\prime}\right)-1\right) G_{\alpha}^{\nu} f\left(x^{\prime}\right) \\
& =\left(m\left(x^{\prime}\right)-1\right) G_{\alpha}^{\nu} f\left(x^{\prime}\right), \quad x^{\prime} \in \Sigma_{2},
\end{aligned}
$$


so that

$$
\begin{aligned}
G_{\alpha} f & =G_{\alpha}^{\nu} f+H_{\alpha}\left(-{\overline{L H_{\alpha}}}^{-1}\left(L G_{\alpha}^{\nu} f\right)\right) \\
& =G_{\alpha}^{\nu}+H_{\alpha}\left(-{\overline{L H_{\alpha}}}^{-1}\left(\left.(m-1) G_{\alpha}^{\nu} f\right|_{\Sigma_{2}}\right)\right)
\end{aligned}
$$

Therefore, by the non-negativity of $H_{\alpha}$ and $-{\overline{L H_{\alpha}}}^{-1}$ it follows that

$$
\begin{aligned}
G_{\alpha} f & =G_{\alpha}^{\nu} f+H_{\alpha}\left(-{\overline{L H_{\alpha}}}^{-1}\left(\left.(m-1) G_{\alpha}^{\nu} f\right|_{\Sigma_{2}}\right)\right) \leq G_{\alpha}^{\nu} f \\
& \leq \frac{1}{\alpha} \max _{\bar{D}} f \quad \text { on } \bar{D}
\end{aligned}
$$

since $\left(m\left(x^{\prime}\right)-1\right) G_{\alpha}^{\nu} f\left(x^{\prime}\right) \leq 0$ on $\Sigma_{2}$ and $\left\|G_{\alpha}^{\nu}\right\| \leq 1 / \alpha$. This proves $\left(7.11^{\prime}\right)$ and hence (7.12).

(iii) Finally we prove the density of $\mathcal{D}\left(\mathfrak{A}_{0}\right)$ in $C_{0}(\bar{D} \backslash M)$. In view of (7.8), it suffices to show that

$$
\lim _{\alpha \rightarrow+\infty}\left\|\alpha G_{\alpha} f-f\right\|_{\infty}=0, \quad f \in C_{0}(\bar{D} \backslash M) \cap C^{\infty}(\bar{D}) .
$$

We recall (cf. (7.4)) that

$$
\begin{aligned}
\alpha G_{\alpha} f-f & =\alpha G_{\alpha}^{\nu} f-f-\alpha H_{\alpha}\left({\overline{L H_{\alpha}}}^{-1}\left(L G_{\alpha}^{\nu} f\right)\right) \\
& =\left(\alpha G_{\alpha}^{\nu} f-f\right)+H_{\alpha}\left({\overline{L H_{\alpha}}}^{-1}\left(\left.\alpha(1-m) G_{\alpha}^{\nu} f\right|_{\Sigma_{2}}\right)\right) .
\end{aligned}
$$

Now we estimate each term on the right of (7.14).

(iii-1) First, applying Theorem 1.1 to the boundary condition $L_{\nu}$ we find from (6.13) that the first term on the right of (7.14) tends to 0 :

$$
\lim _{\alpha \rightarrow+\infty}\left\|\alpha G_{\alpha}^{\nu} f-f\right\|_{\infty}=0
$$

(iii-2) To estimate the second term, we remark that

$$
\begin{aligned}
H_{\alpha}\left({\overline{L H_{\alpha}}}^{-1}\left(\left.\alpha(1-m) G_{\alpha}^{\nu} f\right|_{\Sigma_{2}}\right)\right)= & H_{\alpha}\left({\overline{L H_{\alpha}}}^{-1}\left(\left.(1-m) f\right|_{\Sigma_{2}}\right)\right) \\
& +H_{\alpha}\left({\overline{L H_{\alpha}}}^{-1}\left(\left.(1-m)\left(\alpha G_{\alpha}^{\nu} f-f\right)\right|_{\Sigma_{2}}\right)\right) .
\end{aligned}
$$

However, by (7.7) we have

$$
\begin{aligned}
\| H_{\alpha}\left({\overline{L H_{\alpha}}}^{-1}((1-m)\right. & \left.\left.\left.\left(\alpha G_{\alpha}^{\nu} f-f\right)\right|_{\Sigma_{2}}\right)\right) \|_{\infty} \\
\leq & \left\|-\overline{L H}_{\alpha}^{-1}\right\| \cdot\left\|\left.(1-m)\left(\alpha G_{\alpha}^{\nu} f-f\right)\right|_{\Sigma_{2}}\right\|_{\infty} \\
\leq & \frac{1}{k_{\alpha}}\left\|\left.(1-m)\left(\alpha G_{\alpha}^{\nu} f-f\right)\right|_{\Sigma_{2}}\right\|_{\infty} \\
\leq & \frac{1}{k_{1}}\left\|\alpha G_{\alpha}^{\nu} f-f\right\|_{\infty} \rightarrow 0 \quad \text { as } \alpha \rightarrow+\infty
\end{aligned}
$$

Here we have used the fact that

$$
k_{1}=-\max _{x^{\prime} \in \Sigma_{2}} L H_{1} 1\left(x^{\prime}\right) \leq k_{\alpha}=-\max _{x^{\prime} \in \Sigma_{2}} L H_{\alpha} 1\left(x^{\prime}\right) \quad \text { for all } \alpha \geq 1 .
$$


Thus we are reduced to the study of the term

$$
H_{\alpha}\left({\overline{L H_{\alpha}}}^{-1}\left(\left.(1-m) f\right|_{\Sigma_{2}}\right)\right) .
$$

Now, for any given $\varepsilon>0$, one can find a function $h \in C^{\infty}\left(\Sigma_{2}\right)$ such that $h\left(x^{\prime}\right)=0$ near $M$ and $\left\|\left.(1-m) f\right|_{\Sigma_{2}}-h\right\|_{\infty}<\varepsilon$. Then for all $\alpha \geq 1$ we have

$$
\begin{aligned}
& \left\|H_{\alpha}\left({\overline{L H_{\alpha}}}^{-1}\left(\left.(1-m) f\right|_{\Sigma_{2}}\right)\right)-H_{\alpha}\left({\overline{L H_{\alpha}}}^{-1} h\right)\right\|_{\infty} \\
& \quad \leq\left\|-{\overline{L H_{\alpha}}}^{-1}\right\| \cdot\left\|\left.(1-m) f\right|_{\Sigma_{2}}-h\right\|_{\infty} \leq \varepsilon / k_{\alpha} \leq \varepsilon / k_{1} .
\end{aligned}
$$

Furthermore one can find a function $\theta \in C_{0}^{\infty}\left(\Sigma_{2}\right)$ such that $\theta\left(x^{\prime}\right)=1$ near $M$ and $\left(1-\theta\left(x^{\prime}\right)\right) h\left(x^{\prime}\right)=h\left(x^{\prime}\right)$ on $\Sigma_{2}$. Then

$$
\begin{aligned}
h\left(x^{\prime}\right) & =\left(1-\theta\left(x^{\prime}\right)\right) h\left(x^{\prime}\right)=\left(-L H_{\alpha} 1\left(x^{\prime}\right)\right)\left(\frac{1-\theta\left(x^{\prime}\right)}{-L H_{\alpha} 1\left(x^{\prime}\right)}\right) h\left(x^{\prime}\right) \\
& \leq\left[\max _{x^{\prime} \in \Sigma_{2}}\left(\frac{1-\theta\left(x^{\prime}\right)}{-L H_{\alpha} 1\left(x^{\prime}\right)}\right)\right]\|h\|_{\infty}\left(-L H_{\alpha} 1\left(x^{\prime}\right)\right) .
\end{aligned}
$$

Since the operator $-{\overline{L H_{\alpha}}}^{-1}$ is non-negative on $C\left(\Sigma_{2}\right)$, it follows that

$$
-{\overline{L H_{\alpha}}}^{-1} h \leq \max _{x^{\prime} \in \Sigma_{2}}\left(\frac{1-\theta\left(x^{\prime}\right)}{-L H_{\alpha} 1\left(x^{\prime}\right)}\right)\|h\|_{\infty} \quad \text { on } \Sigma_{2},
$$

so that

$$
\left\|H_{\alpha}\left({\overline{L H_{\alpha}}}^{-1} h\right)\right\|_{\infty} \leq\left\|-{\overline{L H_{\alpha}}}^{-1} h\right\|_{\infty} \leq \max _{x^{\prime} \in \Sigma_{2}}\left(\frac{1-\theta\left(x^{\prime}\right)}{-L H_{\alpha} 1\left(x^{\prime}\right)}\right)\|h\|_{\infty} .
$$

However there exists a constant $c_{0}>0$ such that

Hence

$$
0 \leq \frac{1-\theta\left(x^{\prime}\right)}{m\left(x^{\prime}\right)} \leq c_{0}, \quad x^{\prime} \in \Sigma_{2} .
$$

$$
\frac{1-\theta\left(x^{\prime}\right)}{-L H_{\alpha} 1\left(x^{\prime}\right)} \leq \frac{1-\theta\left(x^{\prime}\right)}{m\left(x^{\prime}\right)\left(-L_{\nu} H_{\alpha} 1\left(x^{\prime}\right)\right)+\left(1-m\left(x^{\prime}\right)\right)} \leq c_{0}\left\|\frac{1}{-L_{\nu} H_{\alpha} 1}\right\|_{\infty} .
$$

In view of Lemma 6.2, this implies that

$$
\lim _{\alpha \rightarrow+\infty}\left[\max _{x^{\prime} \in \Sigma_{2}}\left(\frac{1-\theta\left(x^{\prime}\right)}{-L H_{\alpha} 1\left(x^{\prime}\right)}\right)\right]=0 .
$$

By (7.16) and (7.17), it follows that

$$
\begin{aligned}
\limsup _{\alpha \rightarrow+\infty}\left\|H_{\alpha}\left({\overline{L H_{\alpha}}}^{-1}\left(\left.(1-m) f\right|_{\Sigma_{2}}\right)\right)\right\|_{\infty} \\
\leq \limsup _{\alpha \rightarrow+\infty}\left\|H_{\alpha}\left({\overline{L H_{\alpha}}}^{-1} h\right)\right\|_{\infty} \\
\quad+\left\|H_{\alpha}\left({\overline{L H_{\alpha}}}^{-1}\left(\left.(1-m) f\right|_{\Sigma_{2}}\right)\right)-H_{\alpha}\left({\overline{L H_{\alpha}}}^{-1} h\right)\right\|_{\infty} \\
\leq \lim _{\alpha \rightarrow+\infty}\left[\max _{x^{\prime} \in \Sigma_{2}}\left(\frac{1-\theta\left(x^{\prime}\right)}{-L H_{\alpha} 1\left(x^{\prime}\right)}\right)\right]\|h\|_{\infty}+\frac{\varepsilon}{k_{1}} \leq \frac{\varepsilon}{k_{1}} .
\end{aligned}
$$


Since $\varepsilon$ is arbitrary, this proves that

$$
\lim _{\alpha \rightarrow+\infty}\left\|H_{\alpha}\left({\overline{L H_{\alpha}}}^{-1}\left(\left.(1-m) f\right|_{\Sigma_{2}}\right)\right)\right\|_{\infty}=0
$$

Combining (7.15) and (7.18) we find that the second term on the right of (7.14) also tends to zero:

$$
\lim _{\alpha \rightarrow+\infty}\left\|H_{\alpha}\left({\overline{L H_{\alpha}}}^{-1}\left(\left.\alpha(1-m) G_{\alpha}^{\nu} f\right|_{\Sigma_{2}}\right)\right)\right\|_{\infty}=0 .
$$

This completes the proof of (7.13) and hence of assertion (iii).

$(\mathrm{V})$ Summing up, we have proved that the operator $\mathfrak{A}_{0}$ defined by (7.2) and (7.3) satisfies conditions (a) through (d) of Theorem 2.3. Hence, by $\left(7.1^{\prime}\right), \mathfrak{A}_{0}$ is the infinitesimal generator of a Feller semigroup $\left\{U_{t}\right\}_{t \geq 0}$ on $\bar{D} \backslash M$.

The proof of Theorem 7.1 and hence of Theorem 1.2 is now complete.

Acknowledgements. A major part of this work was done at the University of Bologna and the University of Bari in June, 1998 under the auspices of G.N.A.F.A. of C.N.R. while the first author was on leave from the University of Tsukuba. The research of the first author is partially supported by Grant-in-Aid for General Scientific Research (No. 10440050), Ministry of Education, Science and Culture of Japan.

\section{References}

[1] J.-M. Bony, P. Courrège et P. Priouret, Semi-groupes de Feller sur une variété à bord compacte et problèmes aux limites intégro-différentiels du second ordre donnant lieu au principe du maximum, Ann. Inst. Fourier (Grenoble) 18 (1968), no. 2, 369-521.

[2] C. Cancelier, Problèmes aux limites pseudo-différentiels donnant lieu au principe du maximum, Comm. Partial Differential Equations 11 (1986), 1677-1726.

[3] P. Cattiaux, Stochastic calculus and degenerate boundary value problems, Ann. Inst. Fourier (Grenoble) 42 (1992), 541-624.

[4] J. Chazarain et A. Piriou, Introduction à la théorie des équations aux dérivées partielles linéaires, Gauthier-Villars, Paris, 1981.

[5] E. B. Dynkin, Markov Processes I, II, Springer, Berlin, 1965.

[6] W. Feller, The parabolic differential equations and the associated semigroups of transformations, Ann. of Math. 55 (1952), 468-519.

[7] —, On second order differential equations, ibid. 61 (1955), 90-105.

[8] G. Fichera, Sulle equazioni differenziali lineari ellittico-paraboliche del secondo ordine, Atti Accad. Naz. Lincei Mem. 5 (1956), 1-30.

[9] L. Hörmander, The Analysis of Linear Partial Differential Operators III, Springer, Berlin, 1985.

[10] A. N. Kolmogorov, Über die analytischen Methoden in der Wahrscheinlichkeitsrechnung, Math. Ann. 104 (1931), 415-458.

[11] H. Kumano-go, Pseudo-Differential Operators, MIT Press, Cambridge, MA, 1981.

[12] J. Lamperti, Stochastic Processes, Springer, New York, 1977. 
[13] O. A. Oleŭnik and E. V. Radkevich, Second Order Equations with Nonnegative Characteristic Form, Itogi Nauki, Moscow, 1971 (in Russian); English transl., Amer. Math. Soc., Providence, RI, and Plenum Press, New York, 1973.

[14] K. Sato and T. Ueno, Multi-dimensional diffusion and the Markov process on the boundary, J. Math. Kyoto Univ. 14 (1965), 529-605.

[15] D. W. Stroock and S. R. S. Varadhan, On degenerate elliptic-parabolic operators of second order and their associated diffusions, Comm. Pure Appl. Math. 25 (1972), 651-713.

[16] K. Taira, Diffusion Processes and Partial Differential Equations, Academic Press, San Diego, 1988.

[17] - On the existence of Feller semigroups with Dirichlet condition, Tsukuba J. Math. 17 (1993), 377-427.

[18] —, On the existence of Feller semigroups with boundary conditions II, J. Funct. Anal. 129 (1995), 108-131.

[19] - Feller semigroups and degenerate elliptic operators I, II, Conferenze del Seminario di Matematica, Università di Bari, No. 274, No. 275, 1999.

[20] M. Taylor, Pseudodifferential Operators, Princeton Univ. Press, Princeton, 1981.

[21] H. Triebel, Interpolation Theory, Function Spaces, Differential Operators, Deutscher Verlag Wiss., Berlin, 1978.

[22] A. D. Wentzell [A. D. Ventsel'], On boundary conditions for multidimensional diffusion processes, Teor. Veroyatnost. i Primenen. 4 (1959), 172-185 (in Russian); English transl.: Theory Probab. 4 (1959), 164-177.

Institute of Mathematics

University of Tsukuba

Tsukuba 305-8571, Japan

E-mail: taira@math.tsukuba.ac.jp

Dipartimento di Matematica

Università di Bari

70125 Bari, Italy

E-mail: romans@pascal.dm.uniba.it
Dipartimento di Matematica Università di Bologna 40126 Bologna, Italy E-mail: favini@dm.unibo.it 